\title{
Quantitative structure determination using grazing scattering of fast atoms: Oxygen-induced missing-row reconstruction of Mo(112)
}

\author{
J. Seifert ${ }^{1,2, *}$ and $\mathrm{H}$. Winter ${ }^{1}$ \\ ${ }^{1}$ Institut für Physik, Humboldt-Universität zu Berlin, Newtonstraße 15, 12489 Berlin, Germany \\ ${ }^{2}$ Fritz-Haber-Institut der Max-Planck-Gesellschaft, Faradayweg 4-6, 14195 Berlin, Germany \\ (Received 18 September 2015; revised manuscript received 14 March 2016; published 10 May 2016)
}

\begin{abstract}
We present an extensive study on the structure of oxygen adsorbates on Mo(112) by means of grazing scattering of fast hydrogen and helium atoms and low-energy electron diffraction. For projectile energies less than $2 \mathrm{keV}$, fast atom diffraction provides information on the surface unit cell and on adsorption sites for low coverages. In the classical scattering regime, we employed so-called triangulation techniques where for an azimuthal rotation of the target axial surface channels are identified. From comparison with computer simulation positions of surface atoms can be derived. Aside from the detection scheme of projectile-induced electron emission, we present details for a new variant of triangulation based on the detection of angular distributions of scattered particles. The different sensitivity of the methods to the topmost surface layers allows us to efficiently set up structural models for four adsorbate phases for which contradicting models exist in literature. The $c(4 \times 2)$ phase is revealed to be one step in the formation of a missing-row reconstruction with $p(1 \times 2)$ unit cell. Our studies demonstrate the potential of grazing scattering of fast atoms for quantitative structure analysis.
\end{abstract}

DOI: 10.1103/PhysRevB.93.205417

\section{INTRODUCTION}

The knowledge of the structure of surfaces is essential in fundamental research and technological applications since it affects the properties of physical and chemical processes at surfaces in a decisive manner. Most of the techniques for quantitative structure analysis developed in the last decades are based on scattering of electrons, photons, or atoms/ions [1-4]. Fairly different interaction mechanisms with the nuclei or electron shell of surface atoms result in different properties such as element specificity, sensitivity to low- $Z$ atoms, information depth, need for long-range order or electron conductivity, and radiation damage. The experimental geometry and the nature of the physical principle behind the individual techniques lead to different sensitivities to bond lengths or angles, layer spacings, or local geometry as well as to different accuracies for lateral and perpendicular distances. The large number of parameters in complex surface structures generally requires information from more than a single technique to set up proper models which then can be tested and optimized by algorithms to fit the experimental data. Therefore, the combined application of several methods is needed in general [4-6].

In the last decade, a number of techniques for surface structure analysis have been developed using grazing scattering of fast atoms with energies from 0.3 to few $10 \mathrm{keV}$ [7-11]. Those methods were applied to various atomically flat and well-ordered surfaces comprising metals, semiconductors, insulators, ultrathin films as well as adsorbates of atoms and molecules. The key feature is a high-surface sensitivity since for glancing angles of about $1^{\circ}$ the energy of $\mathrm{keV}$ particles with respect to their normal motion is about $1 \mathrm{eV}$ only. Then atoms are scattered in front of topmost layers of surface atoms with a typical projectile-surface approach of $1 \AA$ A. The use of neutral particles prevents the surface from

*Corresponding author: jseifert@fhi-berlin.mpg.de being charged and avoids the attraction for ions owing to their image charge. Some methods are also sensitive to the positions of topmost hydrogen atoms [12]. The energy of the projectiles in the $\mathrm{keV}$ regime results in an efficient detection of scattered atoms and angular distributions can be recorded within less than a minute with some $10^{5}$ particles impinging on a macroscopic surface area. Therefore, modifications of the surface can be neglected $[13,14]$. Among these techniques, especially fast atom diffraction (FAD) [11,15-22] with de Broglie wavelengths of some $0.001 \AA$ gained considerable interest and motivated numerous studies with respect to FADs phenomenological description [23-36] and utilization for epitaxial growth monitoring $[37,38]$ as well as qualitative and quantitative structure determination [19-21,33,39-45].

In this paper, we demonstrate the power of grazing scattering of fast atoms in structure determination for the formation of a missing-row reconstruction of the $\operatorname{Mo}(112)$ surface after dissociative adsorption of oxygen. In addition to fast atom diffraction, we apply a variant of a triangulation technique which allows one to obtain quantitative structure information with low requirements on modeling of projectilesurface interactions. This method has already been applied in studies on ultrathin films of titanium oxide on $\mathrm{Mo}(112)$ [44] and vanadium oxide on $\mathrm{Au}(111)$ [46] as well as on layers of the amino acid alanine on $\mathrm{Cu}(110)[21,47]$.

The applications of molybdenum and molybdenum oxide in heterogenous catalysis, microelectronics, or corrosion protection has led to considerable interest in the study of oxygen adsorption on molybdenum single-crystal surfaces, as a precursor for molybdenum oxide and other thin oxide films $[48,49]$. Oxygen exposure at elevated temperatures often results in substantial reordering of the atoms of transitionmetal substrates, especially for surfaces with ridge-and-trough structure. These surface modifications include missing or added-row reconstructions, which can be considered as a step in the formation of surface oxide layers. For the missing-row formation, several growth mechanisms have been identified [50,51]. 
For the Mo(112) surface, a number of different superstructure phases upon oxygen exposure were identified by means of low-energy electron diffraction (LEED). For a low dose of oxygen, a $c(2 \times 4)$ superstructure was observed which converts with increasing dose over $p(2 \times 1)$, superposition $c(4 \times 2)+p(1 \times 2)$, and $p(1 \times 2)$ to $p(1 \times 3)$ and $p(2 \times 3)$ at a high dose $[37,42,52-54]$. The latter phases are accompanied by considerable surface reconstructions and are considered as precursor for the formation of a $\mathrm{MoO}_{2}$ layer or represent one-dimensional strings of molybdenum oxide $[49,55,56]$. All structures were prepared at elevated substrate temperatures or by annealing the as-deposited low-temperature films $(T \gtrsim 800 \mathrm{~K})$ which partly exhibit further structural phases with less order [54]. The $p(2 \times 1)$ superstructure has been attributed to a coverage of $\Theta=0.5$ monolayers (ML) and $p(1 \times 2)$ to $\Theta=1 \mathrm{ML}$ in most studies [52-54,57-59].

The Mo(112) surface exhibits close-packed rows in [11 11$]$ direction separated by $4.46 \AA$ in the transverse direction. This results in a variety of different adsorption sites, but the models for the equilibrated surface structures are controversially discussed in literature. Pseudo-threefold-hollow sites have been concluded on the basis of high-resolution electronenergy-loss spectroscopy (HREELS) [58]. Density functional theory (DFT) studies favored short-bridge adsorption sites on the topmost Mo rows for coverages $\Theta \leqslant 0.5 \mathrm{ML}$ and hollow sites for $\Theta \geqslant 1 \mathrm{ML}$. For the $p(1 \times 2)$ phase, the coexistence of different adsorption geometries has been concluded from XPS measurements $[48,60]$, and DFT calculations reported in the same study indicate a higher oxygen coverage of 1.5 ML. From recent LEED and work function measurements, the transition from short-bridge to threefold-hollow sites was concluded to proceed already at a coverage $\Theta=0.25 \mathrm{ML}$ [54].

\section{EXPERIMENTAL SETUP}

In our experiments we have scattered $\mathrm{H}$ and $\mathrm{He}$ atoms with an energy $E=1.3-25 \mathrm{keV}$ from the adsorbate-covered surface under grazing angles of incidence $\Phi_{\text {in }}$ ranging from $0.2^{\circ}$ to $1.8^{\circ}$ as sketched in Fig. 1. The projectile ions and molecules were produced in a Penning ion source and mass analyzed by means of an analyzing magnet. After neutralization in a gas target operated with $\mathrm{He}$ atoms and removal of residual ions by electric field plates, the projectile beam is collimated by three sets of sub-mm slits to a divergence of about $0.01^{\circ}$. The slits serve also as components of two differential pumping stages in order to maintain a pressure in the upper $10^{-11}$ mbar domain in our UHV setup. The collimated beam is directed onto the surface of the adsorbate covered Mo(112) target mounted on a precision manipulator. Oxygen was dosed by backfilling the chamber with $\mathrm{O}_{2}$ using a UHV leak valve. The $\mathrm{Mo}(112)$ single crystal was heated by electron bombardment from the rear with temperature controlled by means of an infrared thermometer outside the chamber.

The angular distribution of scattered projectiles was recorded by means of a two-dimensional (2D) positionsensitive micro-channel-plate detector (MCP) [61] positioned $0.83 \mathrm{~m}$ behind the target. For light projectiles with an energy of $2 \mathrm{keV}$, the detection efficiency is about $50 \%$ resulting from the geometrical open area of the channel plate [62]. Angular distributions and the resulting diffraction patterns

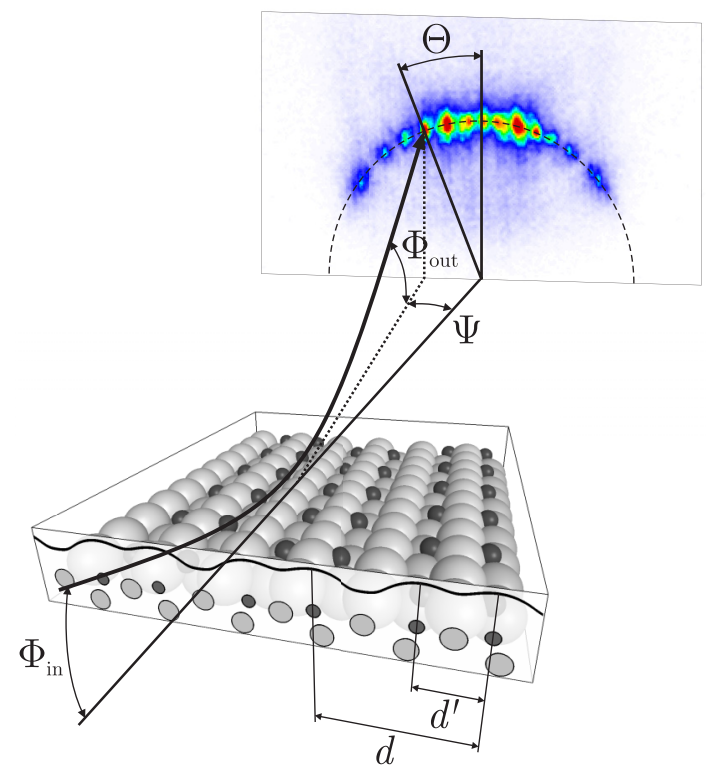

FIG. 1. Sketch of scattering geometry and averaged potential energy surface for $c(2 \times 4) \mathrm{O} / \mathrm{Mo}(112)$ surface.

can be recorded within a few minutes with a low flux of incident particles. Since the maximum counting rate of the complete detection system is limited to some $10^{4}$ counts per second, the equivalent current of incident atoms is in the fA regime. Surface modification caused by atom bombardment can be neglected then. Similar currents are also used for ion beam triangulation where electron number distributions were recorded by means of a surface barrier detector biased to $20 \mathrm{kV}$ $[63,64]$ at a distance of about $6 \mathrm{~cm}$ from the sample.

\section{EXPERIMENTAL METHODS}

For grazing angles of incidence $\Phi_{\text {in }} \sim 1^{\circ}$ scattering proceeds in the regime of "surface channeling" characterized by a fast motion parallel to the surface with energy $E_{\|}=E \cos ^{2} \Phi_{\text {in }}$ and a slow normal motion with energy $E_{\perp}=E \sin ^{2} \Phi_{\text {in }}$. For $\mathrm{keV}$ atoms, $E_{\perp}$ is in the sub-eV domain and the interaction potential has translational symmetry along the fast motion $[7,65]$. For an azimuthal alignment parallel to rows of surface atoms, the effective potential can be regarded as superposition of potentials with axial symmetry for each row. This is known as the regime of "axial surface channeling." The resulting corrugation of the potential surface across the channels leads to out-of-plane scattering denoted by the azimuthal angle $\Psi$ or the deflection angle $\Theta$ in the detection plane (cf. Fig. 1). At the maximum of angular deflection $\Theta_{\mathrm{r}}$, the intensity of scattered atoms is enhanced, the so-called "collisional rainbow" $[66,67]$.

\section{A. Fast atom diffraction}

For sufficiently small angles of incidence and projectile energies, excitations of the solid can be suppressed. Then, quantum coherence is preserved and diffraction patterns can be observed (FAD). The azimuthal angle $\Psi_{n}$ for diffraction spots of order $n$ is related to the lateral periodicity $d_{[h k]}$ of the interaction potential normal to the axial channels formed by strings of surface atoms along the $[h k]$ direction and is given 
by the Bragg relation

$$
d_{[h k]} \sin \Psi_{n}=n \lambda_{\mathrm{dB}}
$$

with $\lambda_{\mathrm{dB}}=h / M v$ being the de Broglie wavelength associated to the center of gravity motion of a particle with mass $M$ and velocity $v, h$ is Planck's constant. The de Broglie wavelength for $1-\mathrm{keV}{ }^{4} \mathrm{He}$ atoms amounts to $0.0045 \AA$, almost three orders of magnitude smaller than the periodicity length $d$ of the interaction potential. Therefore, the resulting splittings between Bragg peaks amount to typically $0.05^{\circ}$ to $0.2^{\circ}$. The angular resolution in the experiment can be made sufficiently large that such small splittings can be resolved.

From the experimental angular splitting between adjacent diffraction spots $\Delta \Psi=\lambda_{\mathrm{dB}} / d_{[h k]}$ one can obtain the symmetry width $d_{[h k]}$ and from comparison with theoretical values for different surface directions the surface unit cell [42]. The averaging of the corrugation of the interaction potential along atomic strings [16] results in the reduction of information on the surface symmetry ${ }^{1}$ to the direction $\boldsymbol{d}_{[h k]}$ perpendicular to the incident beam $\boldsymbol{r}_{[h k]}=h \boldsymbol{b}_{\mathbf{1}}+k \boldsymbol{b}_{\mathbf{2}}$ with $h$ and $k$ being indices of the supercell vectors $\boldsymbol{b}_{\mathbf{1}}$ and $\boldsymbol{b}_{\mathbf{2}}$. The latter are related to the substrate basis vectors $\boldsymbol{a}_{\mathbf{1}}$ and $\boldsymbol{a}_{\mathbf{2}}$ in the matrix notation $M=\left(m_{i j}\right)$ via $\boldsymbol{b}_{\boldsymbol{i}}=m_{i 1} \boldsymbol{a}_{\mathbf{2}}+m_{i 2} \boldsymbol{a}_{\mathbf{2}}$. The symmetry width $d_{[h k]}$ is inversely proportional to the length of the reciprocal lattice vector of the supercell $\boldsymbol{g}_{[h k]}=h \boldsymbol{b}_{\mathbf{1}}^{*}+k \boldsymbol{b}_{\mathbf{2}}^{*}$ with reversed order of indices:

$$
d_{[h k]_{\left(b_{1}, b_{2}\right)}}=\frac{\left|\boldsymbol{b}_{\mathbf{1}} \times \boldsymbol{b}_{\mathbf{2}}\right|}{\left|h \boldsymbol{b}_{\mathbf{1}}+k \boldsymbol{b}_{\mathbf{2}}\right|}=\frac{2 \pi}{\left|\boldsymbol{g}_{[k \bar{h}]}\right|} .
$$

The indices refer to the basis of the superstructure unit cell, indexed by subscripts as $\left[\begin{array}{ll}1 & 2\end{array}\right]=\left[\begin{array}{ll}1 & 2\end{array}\right]_{(1 \times 1)}=\left[\begin{array}{ll}1 & 1\end{array}\right]_{(1 \times 2)}$.

\section{B. Triangulation methods}

Triangulation techniques for grazing scattering of fast ions or atoms are based on the identification of directions with axial surface channeling due to strings of atoms parallel to the particle beam. In former setups, this was realized by recording the current between ground and target which is induced by secondary emission of electrons during the scattering process as a function of the azimuthal orientation of the sample ("ion beam triangulation"). From the resulting "triangulation curves" atomic positions of ultrathin metal and oxide films were derived $[8,68]$. Later, this principle was optimized by the detection of electron number distributions [9,44,69-71], which is discussed below. First, we describe the latest development for the derivation of triangulation curves on the basis of two-dimensional intensity distributions of scattered helium projectiles.

Figure 2(a) shows angular distributions of scattered 2-keV He atoms $\left(\Phi_{\text {in }}=1.3^{\circ}\right)$ from the surface of the $c(4 \times 2)$ oxygen superstructure on $\operatorname{Mo}(112)$ for different azimutal angles $\Gamma$ with respect to the [10] surface direction (panel 3). For normal energies above $E_{\perp} \approx 1 \mathrm{eV}$, the lateral width of diffraction spots gets so large that spots can not be resolved anymore. For

\footnotetext{
${ }^{1}$ Exceptions from this feature of FAD were reported in Ref. [28] where diffraction spots for both transverse and longitudinal directions of reciprocal lattice vectors were observed.
}

He atoms this describes the gradual transition from quantum to classical scattering regime [35]. The angular distributions used for the triangulation techniques can then be regarded as quasiclassical. $^{2}$

For angles a few degrees off low-indexed azimuthal directions, the axial channeling regime persists and the projectiles are also scattered into an annulus in the detection plane but with an enhanced radius corresponding to the enhanced incidence angle with respect to the string of surface atoms and a replaced origin corresponding to the direction of this channel (panel 2). With increasing $\Gamma$, the effective corrugation of the axial channel vanishes and projectiles interact with an averaged "flat" potential. In this regime of planar surface channeling the projectiles are scattered specularly $\Phi_{\text {in }}=\Phi_{\text {out }}$ without azimuthal deflection $\Psi=0$ (panel 1). The resulting intensity distribution does not change for a range of azimuth angles and is referred to "random" scattering direction.

The detection of angular distributions allows one to identify the directions of axial surface channels. The angle of maximal deflection, rainbow angle $\Theta_{\mathrm{r}}$, and the angular range $\Delta \Gamma$ where azimuthal deflection is observed provide information on the width of the axial channel. This information can be reduced to a triangulation curve, where for the individual azimuthal directions the intensity in a small interval of angles around the position of specular reflection [rectangle marked with A in Fig. 2(a) panel 1] is selected and plotted as function of azimuthal angle $\Gamma$ of the target [Fig. 2(b)]. Whenever the incident beam is aligned along axial channels in the surface plane, dips can be observed in the signal of specular intensity. For some axial channels, the exact azimuthal direction can be derived with higher precision from the intensity in the area marked with B [gray curve in Fig. 2(b)].

For an integration time of $10 \mathrm{~s}$ per azimuth angle and computer-controlled rotation of the target in steps of $0.3^{\circ}$ the measurement takes less than one hour. The detection of the whole angular distribution provides an effective normalization of the specular intensity with respect to the total flux of scattered particles. The triangulation curves in Fig. 2(b) have been normalized to 1 . The target has to be aligned very carefully with respect to the axis of rotation prior to the measurement to minimize variations in the polar incidence angle $\left[\Phi_{\text {in }}=1.3^{\circ} \pm 0.1^{\circ}\right.$ in Fig. 2(a)]. For the evaluation of the triangulation curve from the $2 \mathrm{D}$ data, the remaining shift is taken into account by an adjustment of the integration area on the basis of the specular distributions for "random" azimuthal alignments.

In Fig. 2(d), the simulated angular distributions for the same azimuth angles as in Fig. 2(a) are shown for a structural model discussed below. These color-coded plots of intensities are based on several 10.000 trajectories for a three-dimensional motion of projectiles, which are obtained from solving Newton equations of motion using a fourth-order Runge-Kutta method. For the interaction potential, a superposition of interatomic pair potentials in the Thomas-Fermi approach as proposed by

\footnotetext{
${ }^{2}$ For very low polar incidence angles, diffraction spots can be resolved even for 12-keV He atoms for scattering along small axial channels, while for other directions apparently classical distributions were observed.
} 
(a)

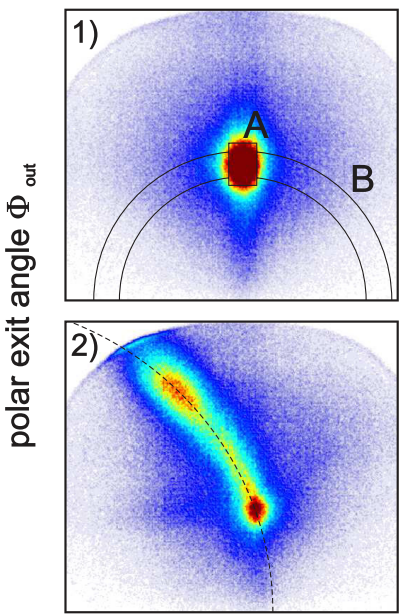

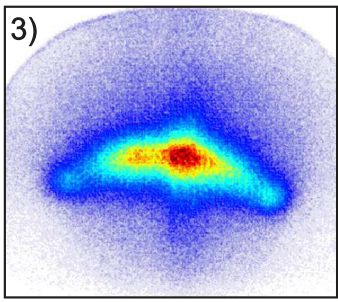

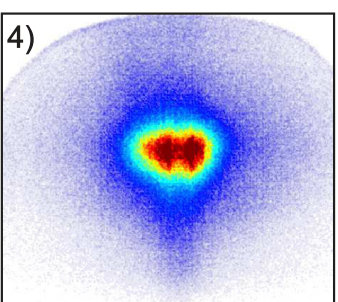

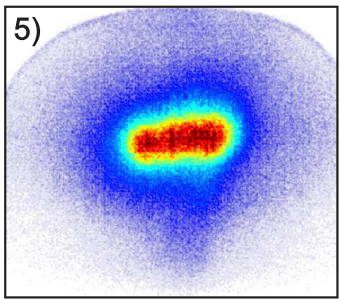

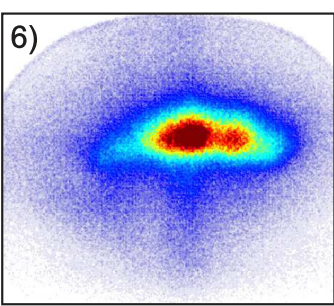

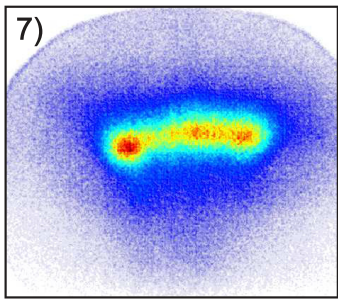

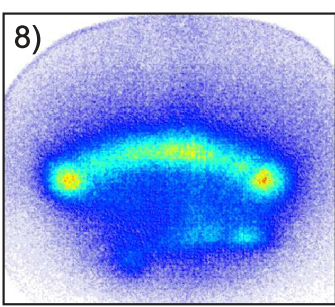

azimuthal exit angle $\Psi$
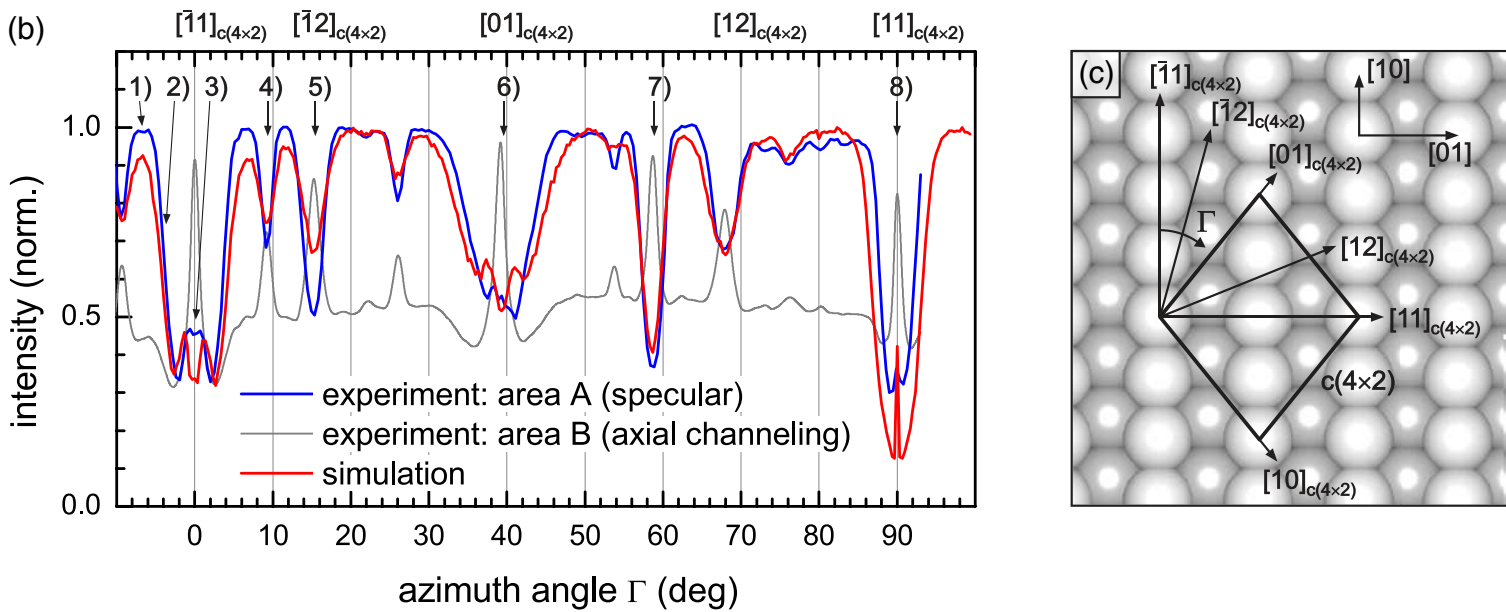

azimuth angle $\Gamma$ (deg)

(d)

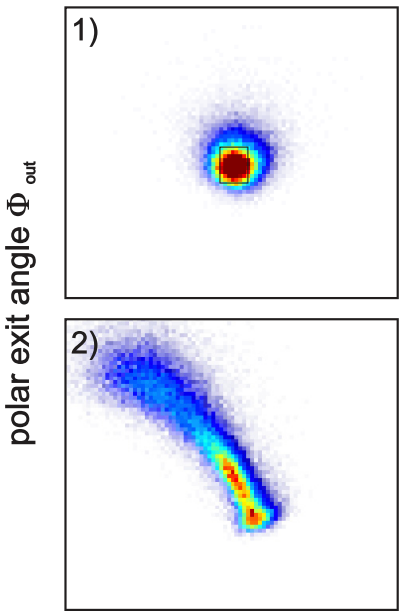

3)

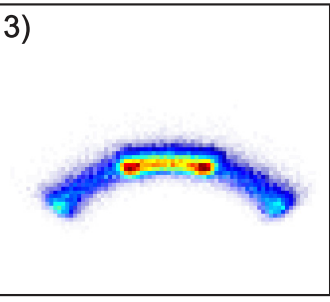

4)

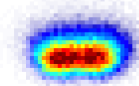

5)

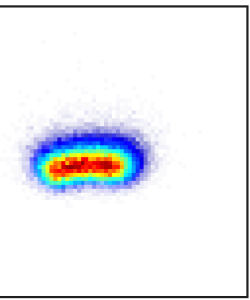

6)

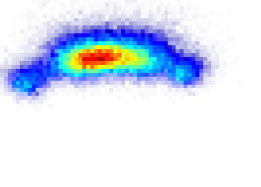

7)

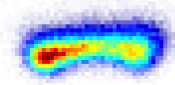

8)

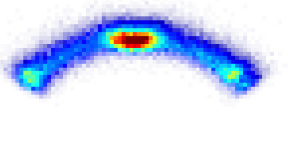

azimuthal exit angle $\Psi$

FIG. 2. Derivation of triangulation curves (b) on the basis of angular distributions of scattered 2-keV helium atoms with polar incidence angle $\Phi_{\text {in }}=1.3^{\circ}$ from the oxygen-covered $\mathrm{Mo}(112)$ surface with $c(4 \times 2)$ symmetry from experiment (a) and simulation (d) for various azimuth angles $\Gamma$.

O'Connor and Biersack [72] was used. Lattice vibrations are taken into account in terms of the Debye model for a frozen lattice and uncorrelated oscillations [73]. The simulated and the experimental angular distributions agree fairly well. For an effective comparison of experiment and simulation for all azimuthal directions $\Gamma$ and a fast verification of structural models triangulation curves can be obtained by extracting the intensity in a small angular range around the direction of specular reflection as performed in the experiment. Since only the positions and relative widths of the axial surface 
channels are relevant, the reproduction of the rainbow angle is not essential. The knowledge of the exact interaction potential is not important since for relatively low normal energies $E_{\perp} \approx 1 \mathrm{eV}$, the corrugation for all axial channels is usually effected in the same way.

Aside from triangulation based on detection of scattered He atoms, also the so-called "ion beam triangulation" was used to study the atomic structure of O/Mo(112). Grazing scattering of $25-\mathrm{keV}$ hydrogen atoms results in a considerable emission of electrons $(\approx 5-10$ electrons per projectile). The number of target electrons emitted per scattering event was detected by means of a surface barrier detector biased to high voltage of about $20 \mathrm{kV}$. Electron emission changes upon azimuthal rotation of the target and by selecting fractions of low electron numbers triangulation curves exhibiting dips for directions of axial channels can be derived. Penetration of projectiles below the surface results in subsurface channeling with a large amount of emitted electrons and therefore the discrimination of events with low electron numbers ensures surface sensitivity $[9,69]$. However, for systems with relatively sparsely distributed topmost surface atoms information from layers deeper than $1 \AA$ can be derived, as demonstrated for the amino acid alanine on $\mathrm{Cu}(110)$ and the vanadyl-terminated $\mathrm{V}_{2} \mathrm{O}_{3}(0001)$ surface $[46,47,74]$. Few, light surface atoms with relatively low scattering cross section and situated well above the substrate atoms affect the projectiles trajectories to a lesser extent and they also do not significantly contribute to the emission of electrons. This resulted in triangulation curves very similar to a clean $\mathrm{Cu}(110)$ or close-packed oxygenterminated $\mathrm{V}_{2} \mathrm{O}_{3}(0001)$ surface, respectively.

For this type of triangulation, electron emission is incorporated in the simulations by summing up probabilities of electronic excitations over complete trajectories. These probabilities are assumed to depend on effective electron densities [75] and thus on the distance of closest approach between fast projectiles and individual target atoms. For the surface with $p(1 \times 2) \mathrm{O} / \mathrm{Mo}(112)$ superstructure we employed the density of valence electrons from DFT calculations for a model from Sierka et al. [48], but the resulting electron number distributions did not satisfactorily describe the experiment, neither with respect to absolute electron yield nor for relative changes for the transition from planar to axial channeling. Hence, we used a summation of adjusted probabilities proportional to $\exp \left(-r^{2} / a\right)$ for the distance $r$ of the projectile to the individual surface atoms. In addition, the probability of a promoted electron to escape towards the vacuum was assumed to decay exponentially with the depth of the present projectile position below the surface. All parameters were adjusted to roughly reproduce the experimentally observed electron number distributions for both planar and axial channeling condition. Then, triangulation curves were derived from the fraction of trajectories resulting in a low number of emitted electrons for each azimuth angle (in steps of $0.33^{\circ}$ ).

Besides the different schemes of detection the two methods differ in the typical energy related to the normal motion of the projectiles $\left(E_{\perp} \approx 1 \mathrm{eV}\right.$ and $\left.E_{\perp} \approx 10 \mathrm{eV}\right)$ and therefore in the average approach to the surface plane $\left(z_{\min } \approx 1 \AA\right.$ and $z_{\min } \approx$ $0.2 \AA$ for $2-\mathrm{keV} \mathrm{He}$ atoms and $25-\mathrm{keV} \mathrm{H}$ atoms, respectively). For grazingly scattered 2-keV $\mathrm{He}$ atoms, trajectories are governed by the (averaged) potential energy surface in front of the surface without penetration which is similar to thermal energy helium atom scattering (HAS) [76]. Conservation of energy results in specular reflection with respect to an axial string of atoms $\Omega_{\text {in }}=\Omega_{\text {out }}$ or the surface plane $\Phi_{\text {in }}=\Phi_{\text {out }}$ (see Ref. [27]) and well-defined angular distributions of scattered particles. In contrast, $25-\mathrm{keV} \mathrm{H}$ atoms are deflected in a much larger angular range and an azimuthal alignment along axial channels results in the possibility of surface penetration and subsurface channeling. A larger polar incidence angle generally increases the approach to the surface but for the regime of axial channeling the probability of penetration, and therefore the relative increase of electron yield, depends also on lower-lying surface atoms within this channel. That means this type of triangulation is more related to the concept of focusing and shadowing in low and medium energy ion scattering $[77,78]$ but with respect to axial channels. For very low incidence angles $\left(\Phi_{\text {in }} \lesssim 0.8^{\circ}\right)$, triangulation curves are mainly determined by the topmost surface layer. In contrast, for scattering of $2-\mathrm{keV} \mathrm{He}$ atoms the incidence angle has a less important role and even for a larger closest approach $z_{\text {min }}$ trajectories are also significantly affected by the tail of interaction potential of lower-lying surface atoms. This major difference of triangulation methods was shown for an ultrathin titanium dioxide film on $\mathrm{Mo}(112)$ [44]. In the Supplemental Material [79], we demonstrate how the atomic positions of different surface layers can be probed by the variation of the incidence angle for the well-studied surface of a monolayer silica on the same substrate [80].

\section{RESULTS AND DISCUSSION}

\section{A. Superstructure phases}

After preparation of the $\operatorname{Mo}(112)$ surface by cycles of grazing angle sputtering with $25-\mathrm{keV} \mathrm{Ar}^{+}$ions and annealing at about $1900 \mathrm{~K}$, molecular oxygen was dosed at a partial pressure ranging from $1 \times 10^{-8}$ to $5 \times 10^{-6}$ mbar at target temperatures from 500 to $1400 \mathrm{~K}$. The adsorbate structure was then studied by LEED and Auger electron spectroscopy (AES) at room temperature. From maxima in the intensity of specularly reflected $\mathrm{He}$ atoms during oxygen exposure the optimal dosages for well-ordered superstructures could be obtained as detailed in Ref. [37]. For not too high temperatures $(<1400 \mathrm{~K})$, it turned out that the oxygen coverage and the resulting surface structure is independent of the specific pressure and time and depends on the dosage only. Therefore, all measurements were performed for an $\mathrm{O}_{2}$ exposure for $80 \mathrm{~s}$ at different partial oxygen pressures. ${ }^{3}$ Furthermore, we showed that all superstructures can not only be prepared by dosing specific amounts of oxygen but also by gradual desorption for annealing at specific temperatures $1300 \mathrm{~K}<T<1500 \mathrm{~K}$. With increasing temperature weakly bound oxygen atoms at different adsorption sites desorb and depending on the remaining oxygen coverage, superstructures evolve in reversed order

\footnotetext{
${ }^{3}$ Oxygen dosages will be given in Langmuir $\left(1 \mathrm{~L}=1.33 \times 10^{-6}\right.$ mbar $\times \mathrm{s})$. The pressure was controlled manually by a leak valve and corrected by the individual base pressure before dosage. For the onset and termination of exposure the pressure changed by more than one order of magnitude within one second.
} 

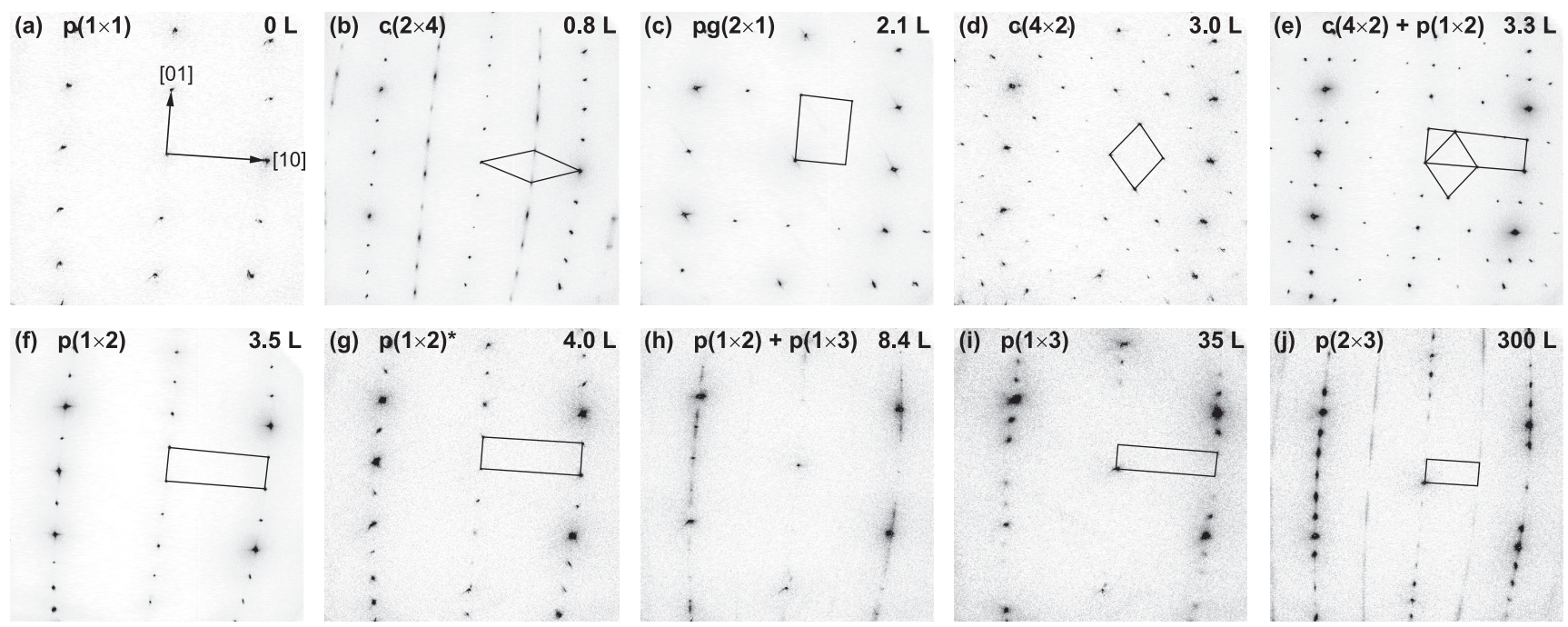

FIG. 3. LEED images $\left(E_{e}=100 \mathrm{eV}\right)$ measured at room temperature for the clean and oxygen-covered Mo(112) surface after exposure to $\mathrm{O}_{2}$ dosages as indicated at a sample temperature of $T=870 \mathrm{~K}$.

of the adsorption process [37]. This includes also structures with pronounced substrate modifications as missing-row reconstructions and molybdenum oxide layers which transform to well-ordered low-coverage adsorbate superstructures upon annealing to $T>1400 \mathrm{~K}$.

In Fig. 3, we show LEED patterns for the clean Mo(112) surface and after dosing 0.8, 2.1, 3.0, 3.3, 3.5, 4.0, 8.4, 35, and $300 \mathrm{~L}$ of $\mathrm{O}_{2}$ at a target temperature of $T=870 \mathrm{~K}$. The patterns for low coverage $(<4 \mathrm{~L})$ are similar to those reported by Fukui et al. [52], however, the diffraction spots shown here are better defined. For the high-coverage phases, the patterns were comparable with those from Refs. [48,55,56]. For the $p(2 \times 1)$ phase emerging at about $2 \mathrm{~L}$ the $\left(h+\frac{1}{2} 0\right)$ spots ( $h$ integer numbers) are missing as observed in previous studies [52] at any electron energy [54]. This is a hint for a glide plane symmetry, i.e., the presence of two mirror-symmetrical halves of the unit cell shifted by half the lattice parameter as we pointed out recently [37] and will be discussed below in the context of FAD. In contrast to Ref. [52], a separate $c(4 \times 2)$ structure without $p(1 \times 2)$ reflexes is observed for $3.0 \mathrm{~L}$, which is followed by a gradual transition from $c(4 \times 2)$ to $p(1 \times 2)$ with both superstructure spots present in LEED. The phase $p(1 \times 2)$ can be found in two different modifications. For a dose of about $4 \mathrm{~L}$ [Fig. $3(\mathrm{~g})$ ] compared to the pattern for $3.5 \mathrm{~L}$ [Fig. 3(f)] the LEED spots of the superlattice are slightly elongated in about $[ \pm 12]$ direction. This modification shows different signatures in triangulation measurements, but it is not investigated here in detail, since it is less ordered. Above $4 \mathrm{~L}$, where the specular intensity decreases rapidly, also the LEED spots are broader. This phase has been denoted as $p(1 \times 2)+p(1 \times 3)$ by Sierka et al. [48]. A $p(1 \times 3)$ phase is present for $35 \mathrm{~L}$ followed by a continuous transition to the $p(2 \times 3)$ phase. The emerging $\left[h+\frac{1}{2} \alpha\right]$ streaks ( $h$ integer numbers and $\alpha$ almost continuous) were attributed to a high amount of antiphase domain boundaries [48,49,56,81]. The dosage for achieving specific superstructures is about a factor of 2 higher than in Ref. [52], but a factor of 2 smaller than in Ref. [48].
We note that all LEED, FAD, and triangulation measurements have been performed at room temperature and that the atomic structures might differ for elevated temperatures. Fedorus et al. [54] showed that the phases described above represent the equilibrated structures, i.e., no further irreversible structural transformations occur upon annealing unless desorption at temperatures exceeding $1300 \mathrm{~K}$. A reversible order-order phase transition is reported only for the lowest coverage from $c(2 \times 4)$ to $p(2 \times 1)$ at $T=730 \mathrm{~K}$. Reversible order-disorder phase transitions were believed to take place at temperatures higher than about $950 \mathrm{~K}$ except from the $c(4 \times 2)$ phase where such a transition is observed at $T=870 \mathrm{~K}$ [54]. These findings agree well with our study using grazing atom scattering [37] where in a temperature range between about 725 (necessary for sufficient ordering) and $1000 \mathrm{~K}$, the surface smoothness for the individual phases did not change much. Therefore, we suppose that for the equilibrated structures and for coverages $\Theta_{\mathrm{O}}>0.25 \mathrm{ML}$ the atomic arrangements derived at room temperature are stable up to 870-950 K before the transition to the disordered "two-dimensional lattice gas" sets in.

\section{B. Surface symmetry from fast atom diffraction}

In order to study the periodicity of the adsorbate superstructures, FAD studies have been performed for all phases of $\mathrm{O} / \mathrm{Mo}(112)$ at room temperature by scattering along various surface directions, and some of the results were presented in Ref. [42]. For the phase $p(1 \times 3)$, considered as precursor for the formation of a $\mathrm{MoO}_{2}$ layer [55,56], the intensity distribution of scattered atoms is very broad pointing to a rough surface. For the phase $p(2 \times 3)$, considered as one-dimensional strings of oxide [49], a very large rainbow angle is observed for scattering in the [10] direction. This implies the presence of a single, broad, symmetrical channel in line with the model presented by Kaya et al. [49]. Due to the large width of the unit cell $d_{[10]}=13.4 \AA$, no Bragg diffraction spots could be resolved with the present setup. 
However, for the phases with a lower amount of oxygen, well-defined diffraction patterns as shown in Fig. 1 were observed [37,42]. The information obtained from elastically scattered projectiles is limited to an annulus in the detection plane with radius $\Phi_{\text {in }}$ and origin at the axis of the surface channel. This intensity from a large number of diffraction patterns recorded for different normal energies can be combined in a so-called "diffraction chart" [11]. In Fig. 4, the diffraction charts for four oxygen adsorbate structures for scattering along three different surface directions are shown. The intensity is color coded and plotted as function of the azimuthal exit angle $\Psi$ and the incidence angle $\Phi_{\text {in }}$ which has been changed in steps of $0.05^{\circ}$. The vertical streaks represent Bragg peaks which reveal a pronounced intensity variation over the entire chart. The total energy $E$ remains unchanged and therefore the positions of individual Bragg peaks are fixed in accordance with Eq. (1). The diffraction charts show a wedge-type arrangement of peaks, where the enhancement of intensity at the rim results from rainbow scattering. The increasing angular width $\Psi_{\max } \approx \Phi_{\text {in }} \sin \Theta_{\mathrm{r}}$ of the diffraction patterns reflects the geometrical enlargement of the intensity distribution for increasing incidence angles $\Phi_{\text {in }}$ and to a lesser extent the enhanced rainbow angle $\Theta_{\mathrm{r}}$ due to an enhanced corrugation of the potential energy surface for increasing normal energies $E_{\perp}=E \sin ^{2} \Phi_{\text {in }}$.

From the spacings of the diffraction peaks $\Delta \Psi$ for the individual direction $[h k]$ the lateral periodicity $d_{[h k]}$ can be determined via the Bragg relation (1). The resulting values
[10]
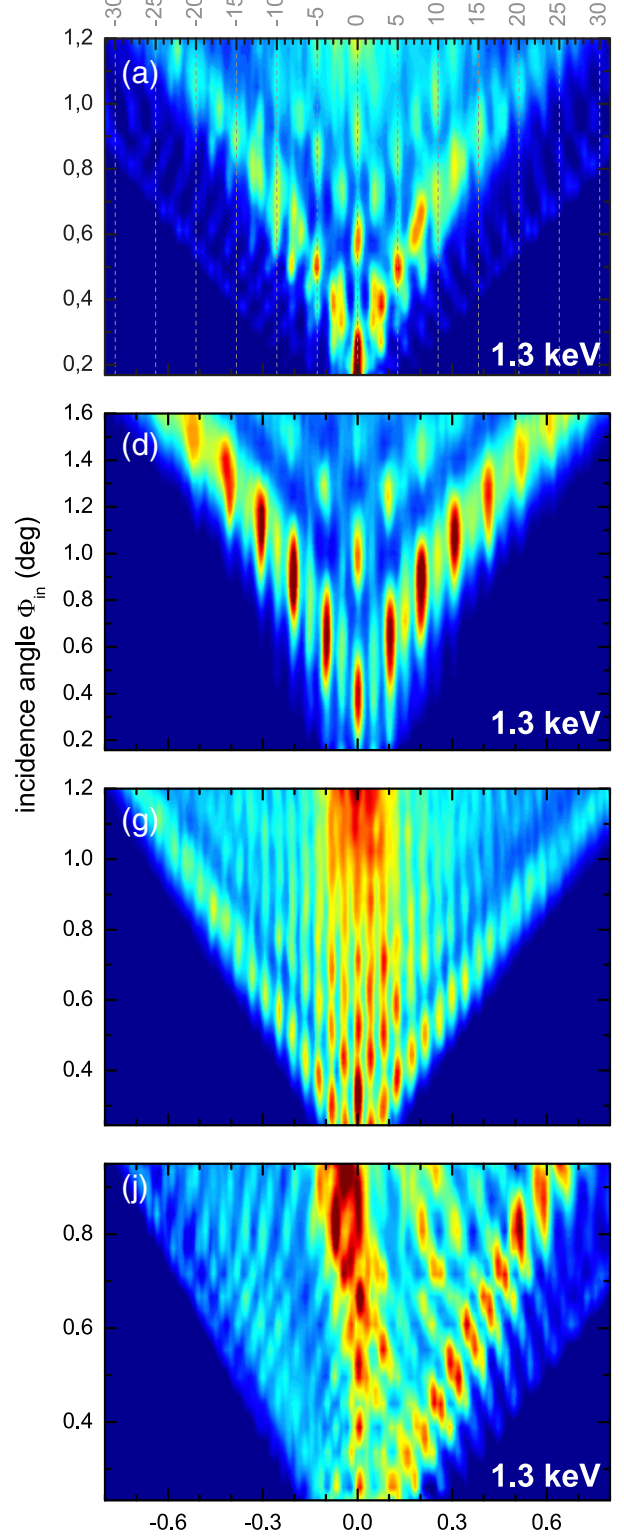

[01]
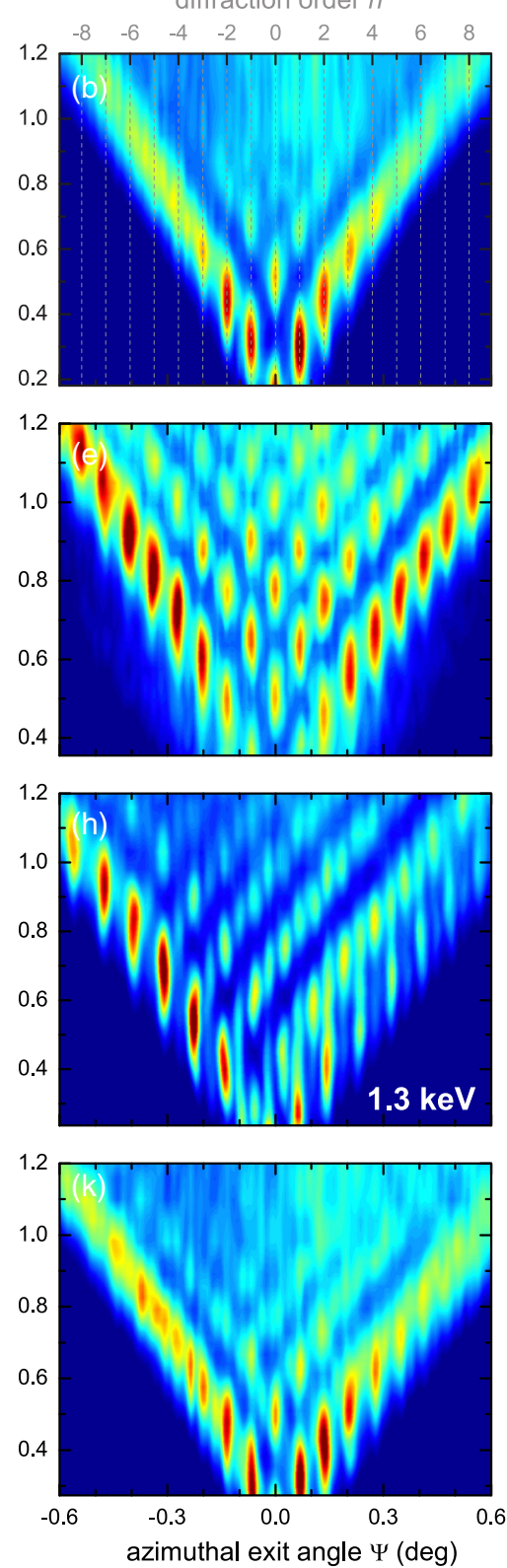

[11]
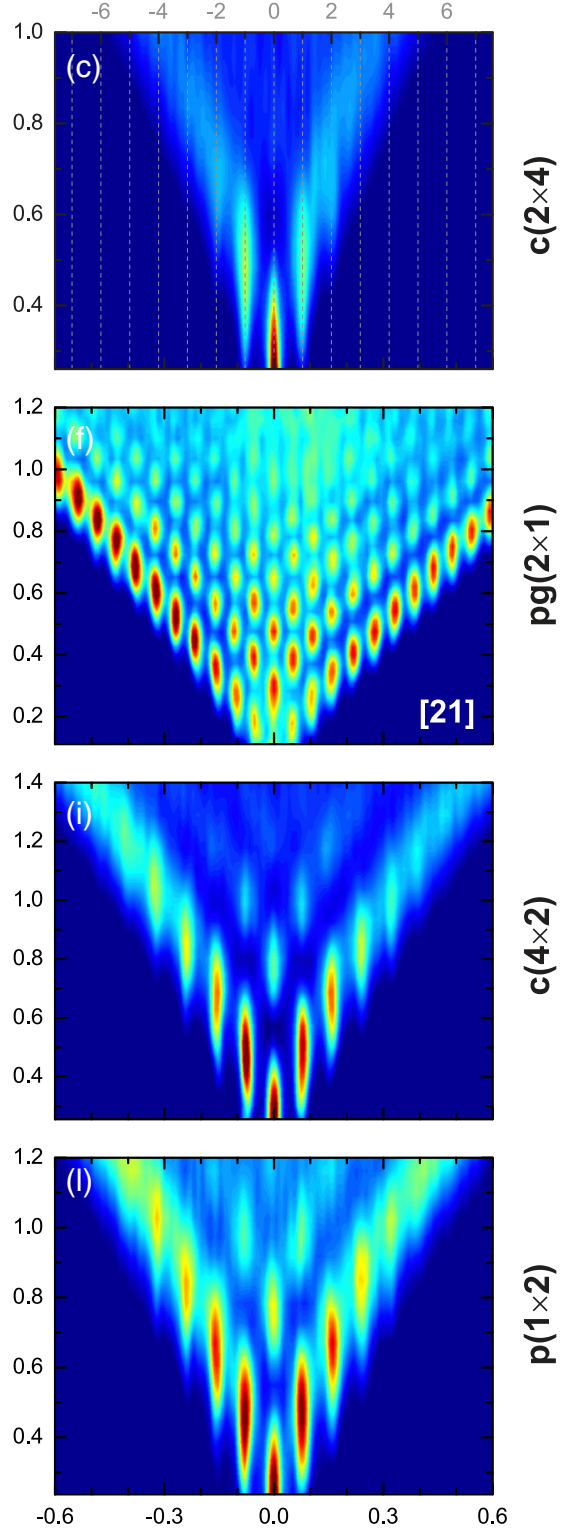

FIG. 4. Diffraction charts based on intensity distributions for various FAD measurements with different polar incidence angles $\Phi_{\text {in }}$ for four oxygen adsorbate structures and scattering along different azimuthal surface directions. If not stated otherwise, helium atoms with $E=2 \mathrm{keV}$ have been used. 


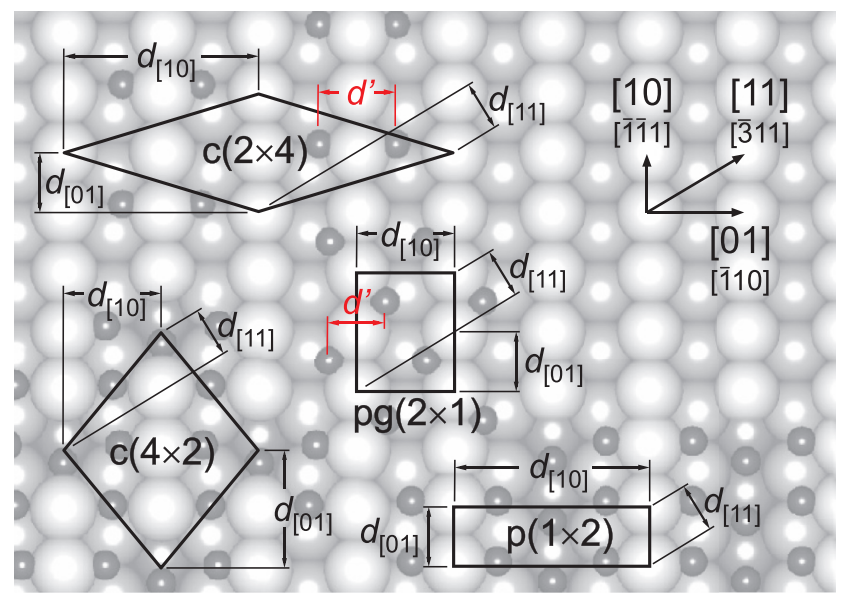

FIG. 5. Unit cell and lateral periodicity $d_{[h k]}$ for scattering in [hk] surface directions for different superstructures on $\mathrm{Mo}(112)$.

agree well with the expected widths for the unit cells as shown in Fig. 5. As pointed out in Ref. [42], the spacings present in the diffraction chart for the phase $p g(2 \times 1)$ along [0 1] direction in Fig. 4(e) correspond to the halved value of the width of $p(2 \times 1)$ unit cell. Therefore, the projected atomic positions in both halves of the unit cell must be identical, which indicates the presence of a glide plane symmetry as observed in the LEED pattern. Thus, this phase must have a coverage of $\Theta_{\mathrm{O}}=1 \mathrm{ML}$, in contrast to $\Theta_{\mathrm{O}}=0.5 \mathrm{ML}$ assumed so far in literature. Since also other superstructure phases were based on this assignment, most of the models in Refs. [52-54,57-59] have to be discarded.

For a single, symmetrically corrugated axial channel with two contributions to the scattering amplitude, the intensities of neighboring diffraction orders are antiphase (for small azimuthal exit angles) [11]. This is the typical situation of FAD and can be observed for all diffraction charts in Fig. 4 except for the panels (a), (d), (g), (h), and (j). For these cases, four different pathways from two separated channels with different corrugation within the unit cell contribute to the scattering amplitude which also results in two different rainbow angles $[20,33,42,44]$. We note that also for one broad single axial channel, more than two pathways are possible due to multiple scattering for a closer approach to the surface mainly at higher normal energies [33,82]. In Figs. 4(h) and 4(j), a slight azimuthal misalignment of the incident beam with respect to the axial channel [27,30-32] leads to a complex and asymmetrical diffraction chart [33]. Such a misalignment is present also in Fig. 4(f), but the intensity modulation for the interference owing to four contributions within the unit cell is much more sensitive to slight changes of the individual pathways.

As demonstrated in detail in Ref. [42], the diffraction charts in Figs. 4(a) and 4(d) for scattering in [10] direction can be compared to a double-slit experiment. The intensity of diffraction spots within the smaller rainbow angle is modulated as function of the azimuthal exit angle $\Psi$ with a periodicity of about 2.5 diffraction orders for the $c(2 \times 4)$ and about 2 diffraction orders for the $p g(2 \times 1)$ structure. This modulation can be described by a double-slit function $\left|G_{d^{\prime}}(\Psi)\right|^{2}=$ $\cos ^{2}\left(\pi d^{\prime} \sin \Psi / \lambda_{\mathrm{dB}}\right)$ which is valid for the whole range of normal energies and has to be added to the scattering amplitude
$I(\Psi) \propto\left|A_{d^{\prime} d}(\Psi)\right|^{2} \cdot\left|G_{d^{\prime}}(\Psi)\right|^{2} \cdot\left|F_{d}(\Psi)\right|^{2}$ containing the form factor $\left|A_{d^{\prime} d}(\Psi)\right|^{2}$ from interference within a single axial channel of width $\left(d-d^{\prime}\right)$ and the structure factor $\left|F_{d}(\Psi)\right|^{2}$ for the lattice with lateral periodicity $d$ (see Fig. 1). The spacing of the "double slits" $d^{\prime}$ can be derived from the periodicity of the additional intensity variation and amounts to $d^{\prime} \approx d / 2.5=$ $3.58 \AA$ and $d^{\prime} \approx d / 2=2.23 \AA$ for the superstructure phases $c(2 \times 4)$ and $p g(2 \times 1)$, respectively. These values compare well with the spacing of strings of oxygen atoms running in [1 0$]$ direction when quasi-threefold-hollow adsorption sites are assumed as also favored in parts of literature (see Fig. 5). While for $c(2 \times 4)$ the relatively large distance $d^{\prime}$ points to the bounding of oxygen atoms to one first-layer and two second-layer molybdenum atoms, it is vice versa for the $p g(2 \times 1)$ adsorbate system. Thus, from simple periodicity considerations, rough estimates on adsorption sites can be derived.

Also, for diffraction charts shown in Figs. 4(h) and 4(j) two different axial channels within the projected lateral periodicity (Fig. 5) result in complex modulation of diffraction intensities, but the small azimuthal misalignment prevents a simple analysis of their width $d^{\prime}$. For all other cases, a single symmetric axial channel is present, and information from within the unit cell requires the detailed analysis of diffraction intensities as a function of normal energy on the basis of proper interaction potentials (usually from $\mathrm{DFT}^{4}$ ) and semiclassical or full quantum theoretical description of interfering matter waves. Calculation of DFT atom-surface potentials for a large number of possible surface structures and their iterative optimization requires an extreme computational effort. Therefore, additional input is necessary before the interferometric nature of FAD with its high intrinsic accuracy can be utilized for quantitative structure determination. This is beyond the scope of this work.

\section{Atomic positions from triangulation}

Quantitative information on atomic positions can be derived from triangulation measurements analyzed by computer simulations. In Fig. 6, the azimuthal scans derived from the number of emitted electrons during scattering of $25-\mathrm{keV} \mathrm{H}$ atoms with polar incidence angle $\Phi_{\text {in }}=1.6^{\circ}$ are compared for the $\operatorname{Mo}(112)$ substrate and all oxygen superstructure phases. As discussed in Sec. III B, for this large incidence angle secondary electron based triangulation measurements for sparsely distributed adsorbate surfaces can provide information on the substrate. For the lowest coverage phases $c(2 \times 4)$ and $p g(2 \times 1)$, the position and order of dips is unchanged compared to the clean surface and only the dip profile is modified, which points to moderate blocking and focusing effects due to few oxygen adatoms. For $c(4 \times 2)$, the nominal coverage is only slightly larger compared to $p g(2 \times 1)$ (oxygen dose is 3.0 and $2.1 \mathrm{~L}$, respectively) but completely new axial channels appear $\left(9^{\circ}\right.$ and $\left.25^{\circ}\right)$ which are not related to low-indexed crystal directions. This points to pronounced

\footnotetext{
${ }^{4}$ With some limitations and modifications also superposition of interatomic pair potentials properly describes atom-surface interaction (see Refs. [38,41,43]).
} 


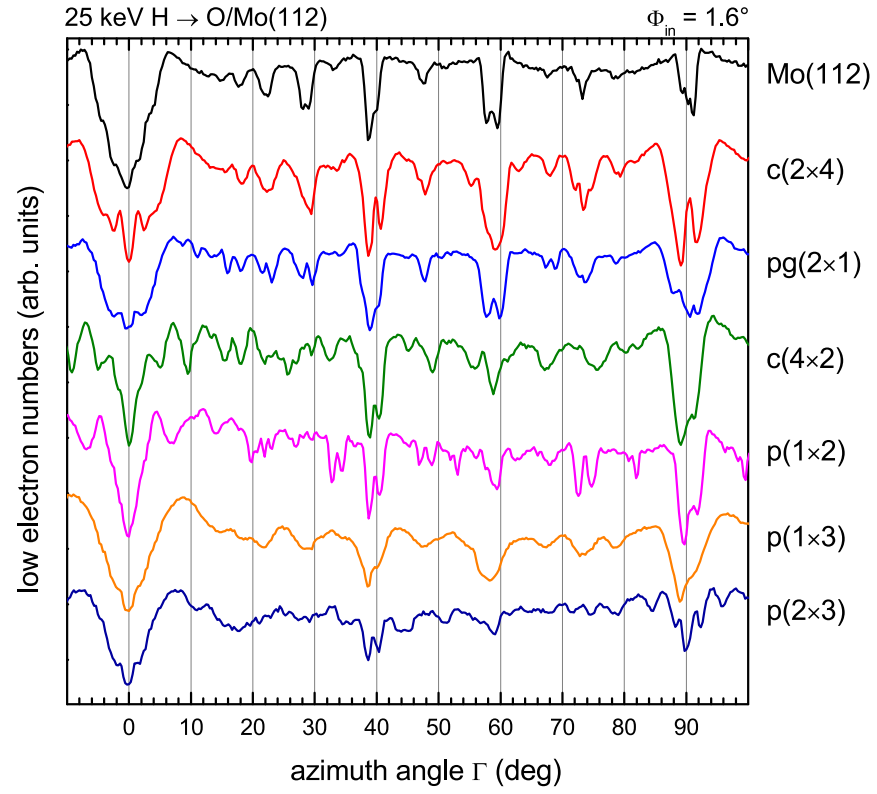

FIG. 6. Triangulation curves based on electron emission during grazing scattering of $25-\mathrm{keV} \mathrm{H}$ atoms from the clean sample and different oxygen superstructures on $\mathrm{Mo}(112)$.

substrate reconstruction which is also present for $p(1 \times 2)$ where for $\Gamma=34^{\circ}$ a prominent channel is present.

The beginning of thin oxide film formation for phase $p(1 \times 3)$ is accompanied by an enhanced surface roughness as can be followed from broader dips. The simulations for the phase $p(2 \times 3)$ on the basis of the models reported from Kaya et al. [49] are not in satisfactory agreement with the experiment (not shown). Due to the complexity of the film, no modifications of atomic positions have been tested. For the phases $c(2 \times 4), c(4 \times 2)$, and $p(1 \times 2)$ triangulation measurements also on the basis of angular distributions of scattered 2-keV He atoms have been realized and will be discussed in the following. All measurements were performed at room temperature.

\section{1. $c(2 \times 4)$}

In the LEED images for an oxygen dosage of about $0.8 \mathrm{~L}$ the spots related to a $c(2 \times 4)$ unit cell differ in form and shape from the spots $p(1 \times 2)$ or even only the latter can be identified. It is likely that the $p(1 \times 2)$ pattern has its origin in a reconstruction of the substrate. As followed in the previous section, the oxygen adatoms are bound in threefold-hollow sites to every second close-packed Mo row in the [1 0] direction, which might cause a slight shift of these substrate atoms in the same direction. For a $c(2 \times 4)$ unit cell and a coverage of $\Theta_{\mathrm{O}}=0.5 \mathrm{ML}$, the oxygen atoms have a spacing of two substrate distances in the [10] direction and therefore an alternating or a pairwise arrangement on each side of the Mo row is possible [Figs. 7(b) and 7(c)]. For the first model, large dips are present in the triangulation curve for azimuth angles $\Gamma=25^{\circ}$ and $47^{\circ}$ [Fig. 7(a)] which corresponds to pronounced axial surface channels for these azimuths. This is in contrast to the experiment and therefore this model can be discarded. At these azimuthal directions, the $c(2 \times 4)$ unit cell has relatively large lateral periodicities as can be seen from the bars in the upper part of Fig. 7(a). A large corrugation of these axial channels seems to be prevented by proper positions of the adsorbate atoms, which is valid for the model with pairwise adsorption sites. Here,

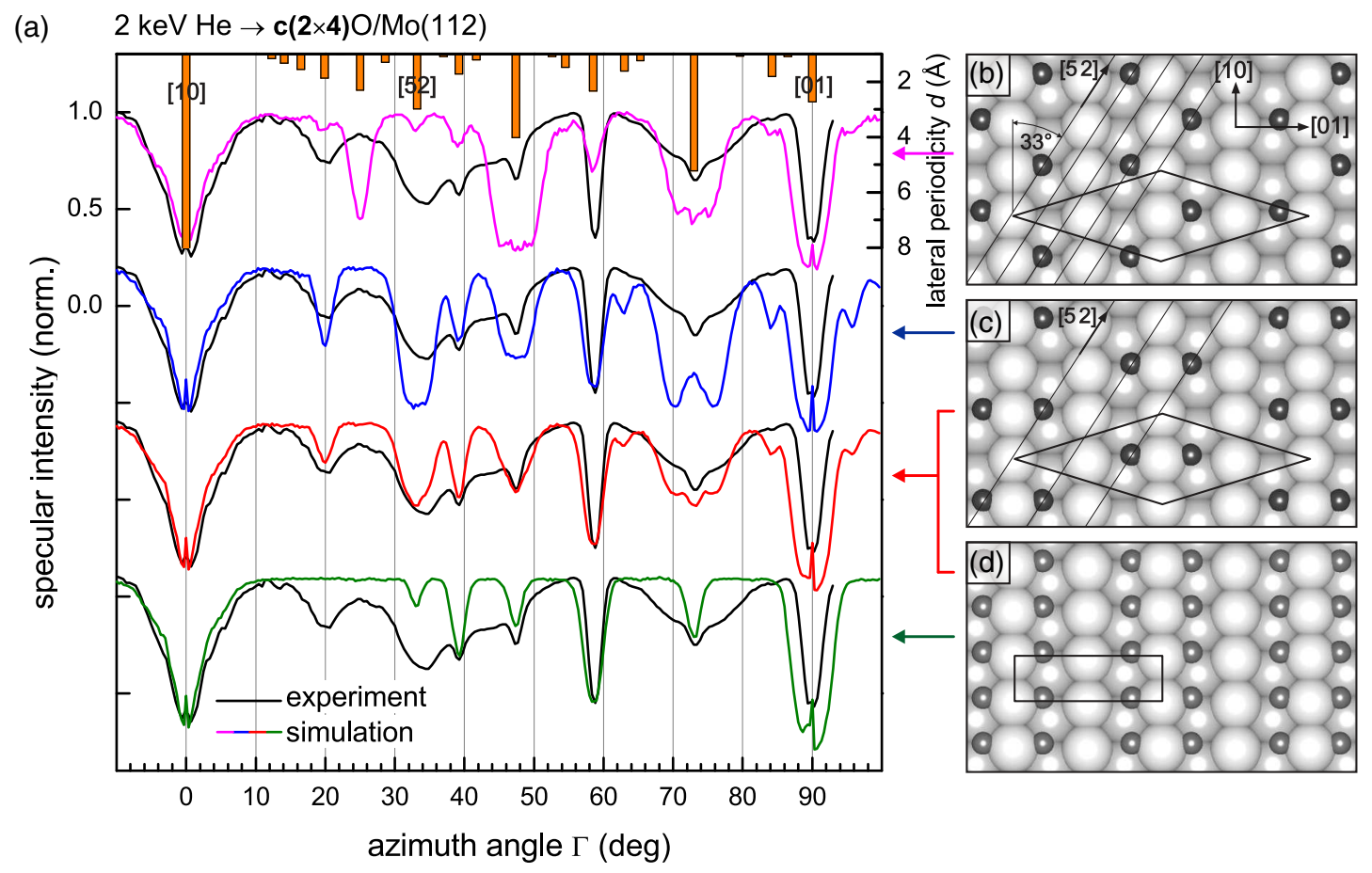

FIG. 7. Triangulation curves based on angular distributions of scattered $2-\mathrm{keV}$ helium atoms from $c(2 \times 4) \mathrm{O} / \mathrm{Mo}(112)$. The lateral periodicities for the individual azimuth angles are illustrated by bars in the upper part of panel (a). The curves in color are simulated triangulation curves based on the models in the right panels. Inclined lines in (b) and (c) visualize strings of oxygen atoms in the [5 2] direction. 
the relative heights of all dips in the triangulation curves are fairly well reproduced and especially the dip for the [52] direction gains considerable intensity due to a doubled spacing of oxygen strings (Fig. 7). However, significant deviations of the absolute heights of the dips are present which could not be eliminated by optimizing the atomic positions. From the elongation of $c(2 \times 4)$ LEED reflexes along [0 1], a high number of antiphase domain boundaries running in the [10] direction can be concluded. We mimic the resulting long-range arrangement by assuming well-ordered $c(2 \times 4)$ domains and areas with a random possession of pairwise adsorption sites. Since one projectile interacts with many surface atoms, the second arrangement can be realized in the simulation by a doubled coverage and a reduction of the interaction potential of the oxygen adatoms by one half [Fig. 7(d)]. The superposition (red curve) with equal weights of the resulting triangulation curve (green) with that from Fig. 7(c) (blue) leads to good agreement with the experimental data.

In literature, short-bridge adsorption sites were discussed for the low-coverage regime. For the models with $\Theta=$ $0.25 \mathrm{ML}$ and one oxygen atom at every second bridging site on top of every second molybdenum ridge, the axial surface channels would have a much higher corrugation. To compensate the resulting large rainbow angles in the simulation the interaction potential would have to be adjusted considerably. When doing so, the triangulation curve (not shown) exhibits dips with the same relative heights as the lateral periodicities of the $c(2 \times 4)$ unit cell [bars in Fig. 7(a)]. This is expected for a single topmost atom in the unit cell, but is clearly in contrast to the experiment.

Figure 8 shows the azimuth scans based on electron emission for three different angles of incidence. For larger $\Phi_{\text {in }}$ there are dips corresponding to a rectangular arrangement of atoms as for the clean $\mathrm{Mo}(112)$ surface unit cell. For a

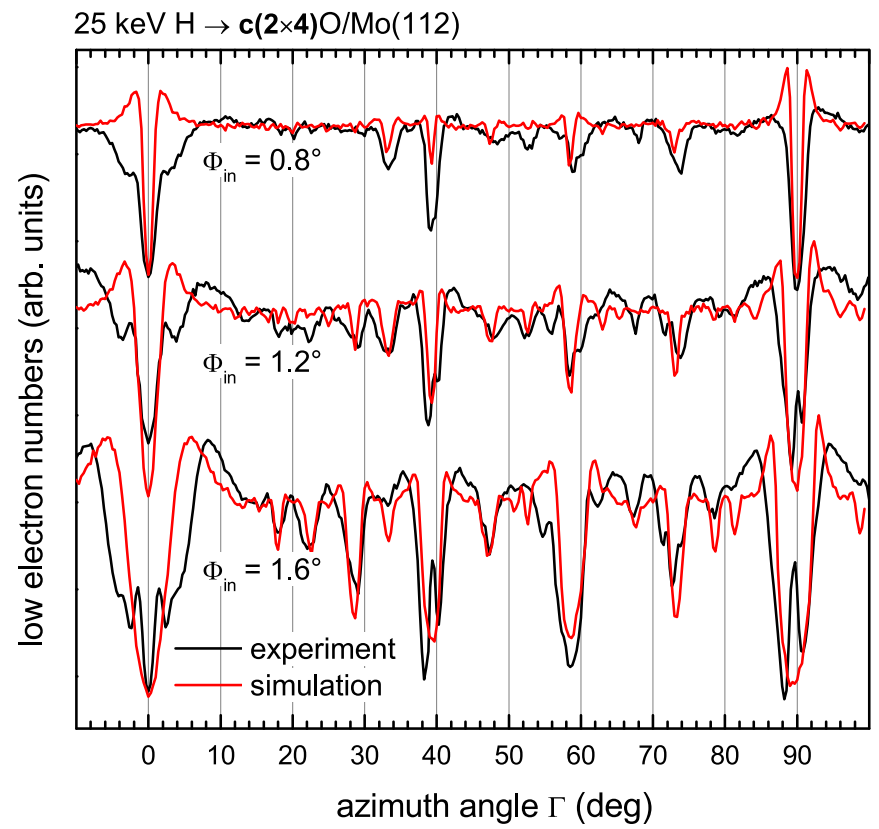

FIG. 8. Triangulation curves from electron emission for $c(2 \times 4) \mathrm{O} / \mathrm{Mo}(112)$ and for different polar incidence angles $\Phi_{\text {in }}$. The simulated curves (red) are based on the superposition of the models shown in Figs. 7(c) and 7(d). reduction of the incidence angle, the sensitivity to the sparsely distributed adsorbate atoms increases. In contrast to the overall decreasing dip heights, a prominent dip for the azimuth $\Gamma=33^{\circ}$ corresponding to the [52] surface direction appears. This characteristic feature as well as most of the other axial channels are being reproduced by the trajectory simulations based on the model in Fig. 7(c), but the differences in the curves between Figs. 7(b) and 7(c) are small for this type of triangulation method.

For the shown structural models and triangulation curves, the atomic positions have been modified until a satisfying agreement of experiment and simulation is achieved. For the oxygen adsorption sites, the following parameters could be deduced where for the ordered [Fig. 7(c)] and random structure [Fig. 7(d)] the same positions have been assumed. The oxygen atoms are separated from the Mo row by $(1.85 \pm 0.1) \AA$ in [0 1] direction and shifted by $(0.35 \pm 0.1) \AA$ in $[10]$ direction from the position directly beside the Mo atom. Presuming that the adsorbate atom has the same distance to the Mo atoms of the second layer, a shift of the Mo row to which oxygen atoms are bound of $(0.1 \pm 0.1) \AA$ in the [1 0] direction follows. ${ }^{5}$

\section{2. $p g(2 \times 1)$}

From the verification of a glide plane symmetry on the basis of LEED and FAD measurements, a coverage of $\Theta_{O}=1 \mathrm{ML}$ is evident for the $p g(2 \times 1)$ structure. From the additional periodicity in the FAD patterns, a smaller distance of the oxygen strings in the [1 0] direction compared to the $c(2 \times 4)$ structure could be deduced. With the resulting adsorption sites, the atomic structure is determined qualitatively as shown in Fig. 9(b). In the left panel of this figure, the experimental and simulated triangulation curves based on electron emission are compared for an incidence angle of $\Phi_{\text {in }}=1.6^{\circ}$. All signatures are well reproduced after the atomic positions have been optimized. The oxygen atoms are located at a distance of $(1.3 \pm 0.2) \AA$ from the Mo rows off the intermediate position of two adjacent Mo atoms. These topmost Mo atoms are rearranged by $0.45 \AA$ in the [10] direction to possess the centered position of the fourfold-hollow sites of the second layer.

\section{3. $c(4 \times 2)$}

In Figs. 10(a) and 11, the triangulation curves resulting from the angular distributions of 2-keV He atoms and from electron emission for scattering of $25-\mathrm{keV} \mathrm{H}$ atoms, respectively, are compared with the corresponding simulated curves. In both cases, we find pronounced dips for azimuth angles $9^{\circ}, 39^{\circ}$, and $68^{\circ}$ (see also Fig. 6 for $\Phi_{\text {in }}=1.6^{\circ}$ ). These channels are not blocked by adsorbate atoms and, therefore, it is possible to probe the arrangement of substrate atoms by means of secondary electron based triangulation with large incidence angles (cf. Sec. III B). The azimuths mentioned correspond to [10 1], [2 1], and [23] surface directions with rather small axial channels for the unreconstructed surface and, consequently,

\footnotetext{
${ }^{5}$ For the unreconstructed $\mathrm{Mo}(112)$ surface, the topmost atoms are $0.455 \AA$ away from the centered hollow site of the second layer. This value is reduced by $0.05 \AA$ for the relaxed surface (Ref. [84]).
} 
(a)

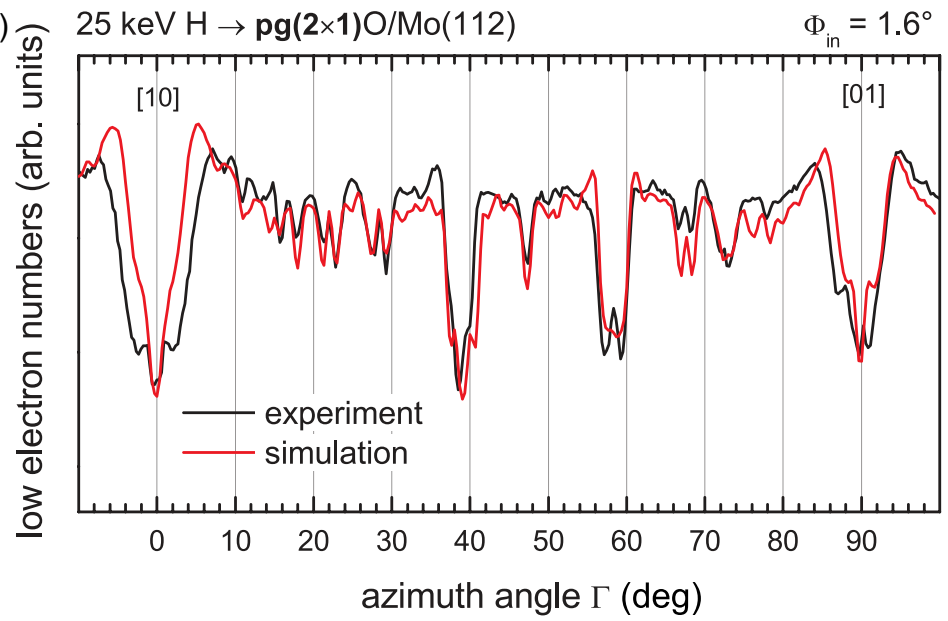

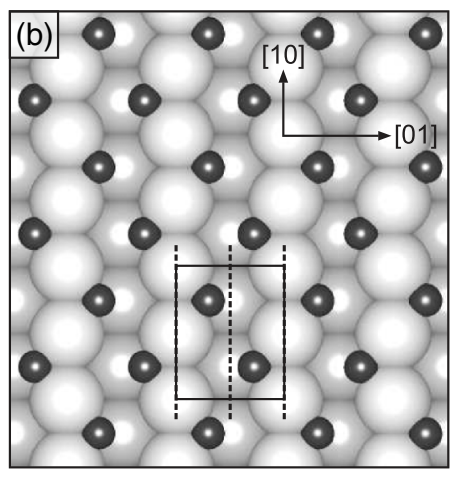

FIG. 9. Triangulation curves from electron emission for $p g(2 \times 1) \mathrm{O} / \mathrm{Mo}(112)$ for experiment (black) and simulation (red) based on the model shown in panel (b). The dashed lines indicate the glide plane symmetry.

small dips would result. However, for the $c(4 \times 2)$ unit cell, the lateral periodicities are relatively high [see bars in Fig. 10(a)] which is in line with the observed large dips and, therefore, it is likely that also the substrate is reconstructed according to this unit cell. This assumption is supported by the need of a higher number of adsorption sites for the enhanced coverage of $\Theta_{\mathrm{O}}=1.25 \mathrm{ML}$ which follows from the intensity oscillation during adsorption [37] and the linear increase of coverage as function of oxygen dose in this regime [52].

In the structural models in Fig. 10, one Mo atom in the unit cell has been removed. Only for this reconstruction of the surface the pronounced signals for the mentioned azimuthal directions are present in the triangulation curves. The exact heights of the dips are determined by the positions of the adsorbate atoms. With the two different threefold-hollow sites discussed so far, two different structural models are possible.
Model A has hollow adsorption sites on both sides of the middle Mo atom and bridge sites between adjacent Mo strings. In model B, four oxygen atoms are adsorbed at both sides of the three adjacent Mo atoms. For both models, a further oxygen atom is located in a deeper layer but the techniques of grazing atom scattering are not very sensitive to its position. For the technique using the angular intensity distributions, the optimization procedure led to good agreement of experimental and simulated triangulation curves for both structural models [Fig. 10(a)]. However, on the basis of electron emission, model $\mathrm{B}$ can be discarded since in contrast to the experiment there are large dips present in the simulated curve for $\Gamma=22^{\circ}$ and $54^{\circ}$ also for larger variations of atom positions (Fig. 11). Good agreement between experiment and simulation is achieved for both triangulation methods when the same positions of oxygen atoms in hollow sites are assumed as for the $c(2 \times 4)$ model (a) $2 \mathrm{keV} \mathrm{He} \rightarrow \mathrm{c}(4 \times 2) \mathrm{O} / \mathrm{Mo}(112)$

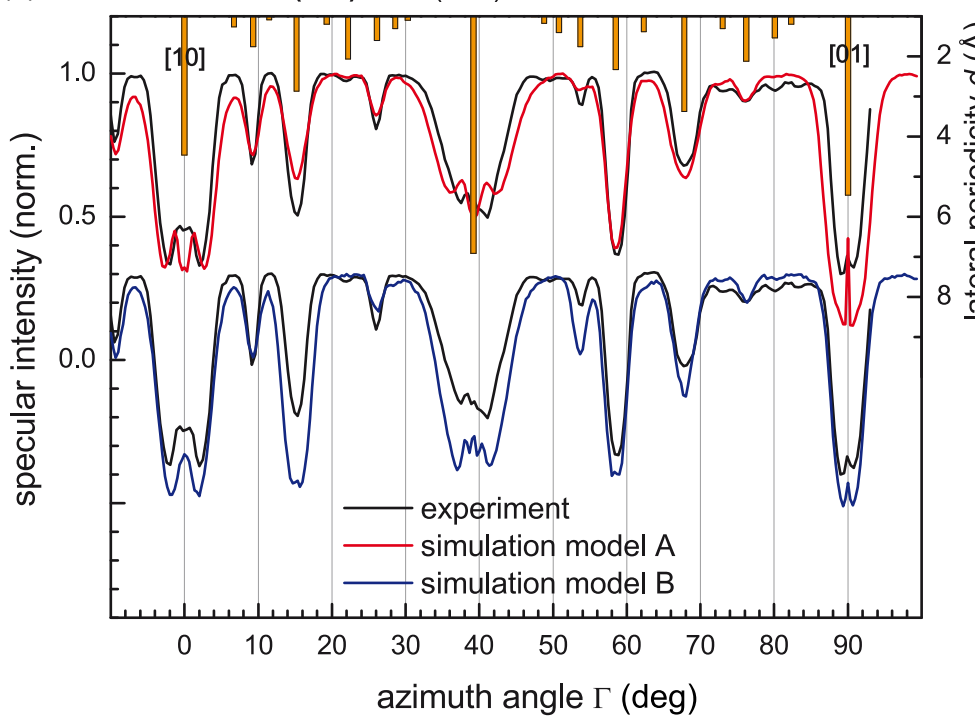

(b)

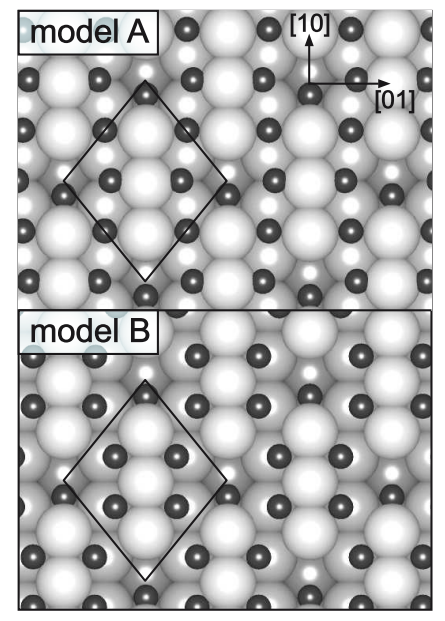

FIG. 10. Triangulation curves based on angular distributions of scattered $2-\mathrm{keV}$ helium atoms $\left(\Phi_{\text {in }}=1.3^{\circ}\right)$ from $c(4 \times 2) \mathrm{O} / \mathrm{Mo}(112)$. The lateral periodicities for the individual azimuth angles are illustrated by bars in the upper part of panel (a). The colored curves are simulated triangulation curves based on the models in the right panels. 


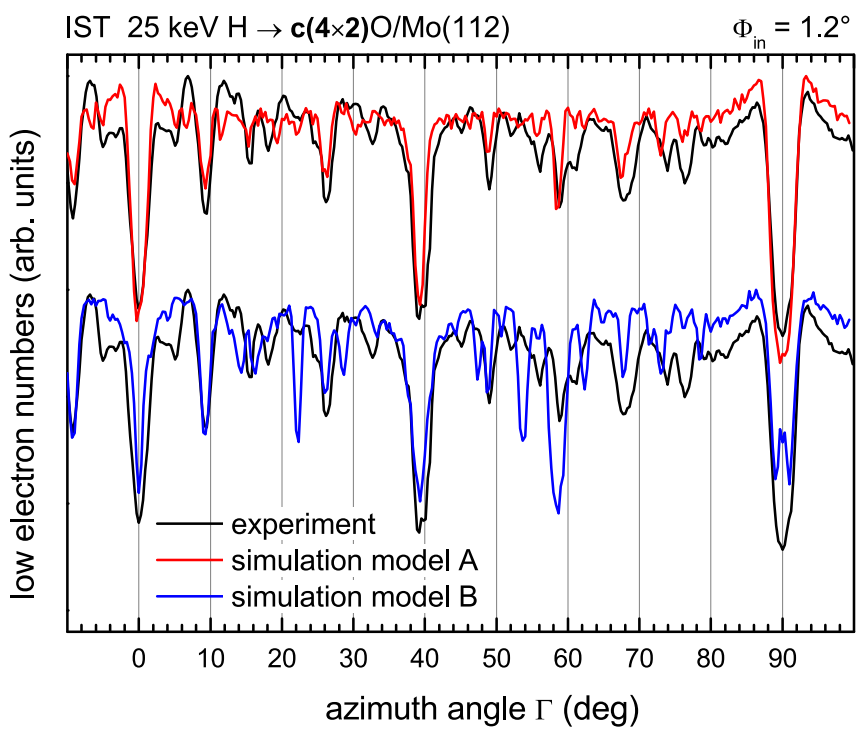

FIG. 11. Triangulation curves from electron emission for $c(4 \times 2) \mathrm{O} / \mathrm{Mo}(112)$. The simulated curves (colored) are based on the models shown in Fig. 10(b).

[distance to the Mo string $(1.85 \pm 0.1) \AA$ ]. The three topmost molybdenum atoms in the unit cell are located at close to centered positions of the fourfold-hollow sites of the second layer and might have a slightly enhanced spacing.

\section{4. $p(1 \times 2)$}

Recent studies using STM and DFT point to a "missingrow" reconstruction for the $p(1 \times 2)$ oxygen superstructure $[48,55,60]$ which seems to be plausible for an oxygen coverage of $\Theta_{\mathrm{O}} \approx 1.5 \mathrm{ML}$. In the triangulation curve for this system in Fig. 12(a) the triple peak signature for the [10] channel also points to this kind of reconstruction. Here, a focusing effect in-between the broad axial channel leads to a blocking of penetration into deeper layers and consequently to maxima in the triangulation curve for
$\Gamma= \pm 3^{\circ}$. The simulation is not able to reproduce this feature. The assumptions on the electron density distribution and electron emission process seem to be not appropriate for channeling in deeper surface layers.

For $\Gamma=33^{\circ}$ ([52] direction) a pronounced dip is present in the triangulation curve. Since also for the $c(2 \times 4)$ phase this characteristic dip was observed a similar adsorption site can be concluded for $p(1 \times 2)$, namely, binding to one Mo atom of the first and to two atoms of the second layer. Compared to $c(2 \times 4)$, the dip for this direction is even more pronounced, which can be explained by the missing molybdenum atoms. The arrangement of topmost adsorbate atoms opens an entrance channel, but further penetration of projectiles would be blocked by the underlying molybdenum atoms of every second row [see Fig. 12(b) in comparison with Fig. 7]. Thus, this provides further evidence for a missing-row reconstruction. Simulations for a number of adsorbate configurations finally led to excellent agreement with the experiment for parameters of the model shown in Fig. 12(b). The oxygen atoms are separated by $(2.03 \pm 0.08) \AA$ from the Mo rows and are displaced by $(0.1 \pm 0.1) \AA$ in [1 0 ] direction from the position directly beside the Mo atoms. Therefore, the oxygen atoms have a more lateral position with respect to the Mo atoms compared to the model for $c(2 \times 4)$. For the derived height $(0.4 \pm 0.15) \AA$ of the oxygen layer above the atoms of the molybdenum ridge, a binding length O-Mo of $(2.05 \pm 0.1) \AA$ follows. For the other oxygen phases, $(1.95 \pm 0.1) \AA ̊$ has been derived. These values compare well with the results from DFT calculations in combination with a genetic algorithm from Sierka et al. [48,60] for their favored model B: projected distance $\mathrm{O}-\mathrm{Mo}$ in [10] direction $0.09 \AA$, in [01] direction $1.97 \AA$, and normal to the surface $0.38 \AA$. The resulting binding length is $2.00 \AA$. Similar as for $c(4 \times 2)$, the triangulation techniques are hardly sensitive to the positions of the oxygen atoms in the trough. We want to note that the errors stated for atom positions are conservative estimates and include uncertainties inherent in the interaction potential.

Figure 13(a) shows the triangulation curves based on the detection of scattered projectiles. For azimuthal angles $\Gamma=9^{\circ}$,
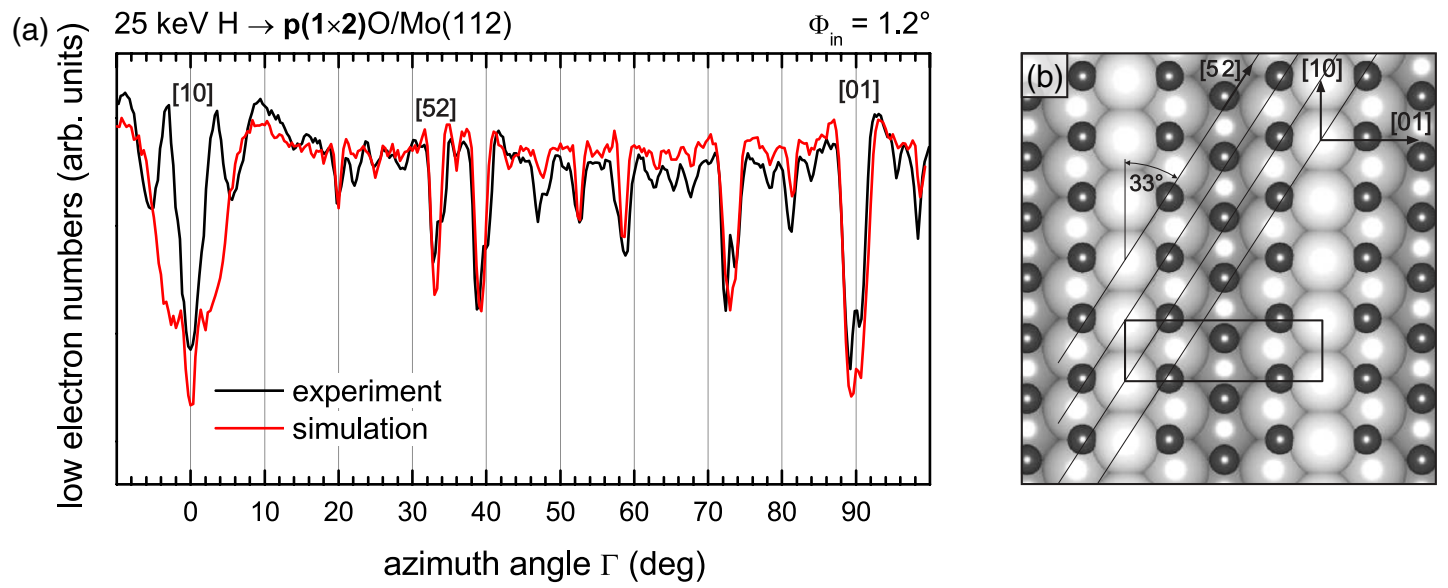

FIG. 12. Triangulation curves from electron emission for $p(1 \times 2) \mathrm{O} / \mathrm{Mo}(112)$ from experiment (black) and simulation (red) based on the model shown in panel (b) which is very similar to the model B derived by Sierka et al. [48]. Inclined lines visualize strings of oxygen atoms in the [5 2] direction. 
(a) $2 \mathrm{keV} \mathrm{He} \rightarrow \mathrm{p}(\mathbf{1} \times \mathbf{2}) \mathrm{O} / \mathrm{Mo}(112)$

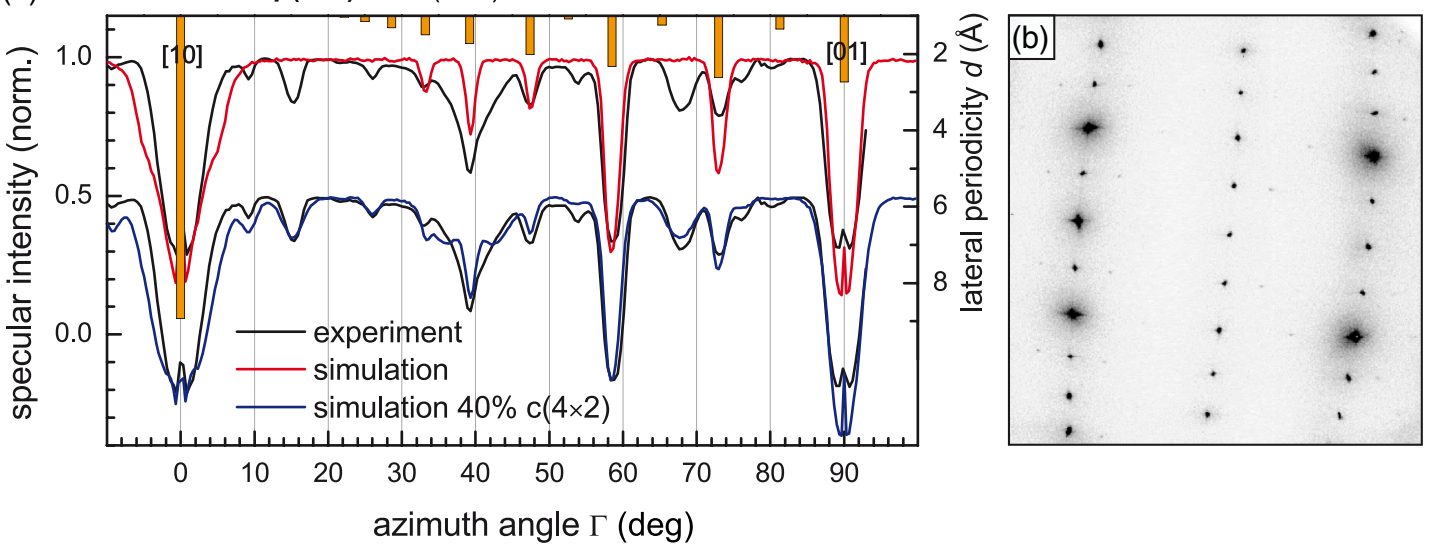

FIG. 13. Triangulation curves based on angular distributions of scattered $2-\mathrm{keV}$ helium atoms from $p(1 \times 2) \mathrm{O} / \mathrm{Mo}(112)$. The lateral periodicities for the individual azimuth angles are illustrated by bars in the upper part of panel (a). The best agreement of experiment and simulation is achieved for the model in Fig. 12(b) with a contribution of $40 \%$ of the surface with $c(4 \times 2)$ superstructure. This is justified by the faint $c(4 \times 2)$ reflexes in the LEED image (panel $\mathrm{b}$ ) for the adsorbate surface for which the triangulation measurement has been performed.

$15^{\circ}, 25^{\circ}$, and $68^{\circ}$, we observe dips in the experimental curve which are not present in the simulation. The comparison with the bars in the upper part of the panel indicating the lateral periodicity of the unit cell for each direction shows that no broad axial channels can be expected at these azimuth angles for a $p(1 \times 2)$ unit cell. A closer look on the LEED pattern for the adsorbate structure at which this triangulation measurement has been performed reveals the presence of domains with $c(4 \times 2)$ unit cell. From the intensity of the LEED reflexes, a relatively small fraction of these domains can be estimated. However, a superposition of the triangulation curves of $60 \% p(1 \times 2)$ and $40 \% c(4 \times 2)$ resulted in good agreement with the experimental curve [Fig. 13(a)]. Regardless of the present composition of superstructure phases, the individual domains must be larger than about $50 \AA$, which is a typical range of a trajectory with significant projectile-surface interaction. From the very sharp superstructure spots in LEED images, an even larger coherence length can be estimated. This holds for all compositions of superstructures for the gradual transition between $c(4 \times 2)$ and $p(1 \times 2)$ regardless of preparation by dosing about $3.3 \mathrm{~L}$ at $870 \mathrm{~K}$ or by desorption of oxygen species from the saturated surface for annealing at about $1350 \mathrm{~K}$. Only after oxygen exposure at temperatures below $750 \mathrm{~K}$ larger LEED spots were observed, but also with both superstructure patterns present. This means for sufficient surface mobility adsorbate and substrate atoms arrange in domains with either $c(4 \times 2)$ or $p(1 \times 2)$ unit cell and the individual atomic structures with oxygen coverages $\Theta_{\mathrm{O}}=1.25 \mathrm{ML}$ and $\Theta_{\mathrm{O}}=1.5 \mathrm{ML}$, respectively, are energetically favorable compared to arrangements with intermediate coverages. This thermodynamic stability caused by the subtle interplay between energy gain for O-Mo bond forming and expense for Mo-Mo bond breaking should be verified by means of density functional theory which was not performed so far for a $c(4 \times 2)$ unit cell. For a detailed understanding of the kinetic mechanisms for the nucleation and growth of $p(1 \times 2)$ domains including the mass transport involved in substrate reordering, further studies are necessary as were presented recently for the missing-row restructuring of $\mathrm{O} / \mathrm{Cu}(110)$ by means of STM and DFT [83].

\section{SUMMARY}

Atomic positions of oxygen superstructures on the Mo(112) surface have been investigated by means of grazing scattering of atoms in the keV energy regime and LEED. For annealing temperatures above $750 \mathrm{~K}$, several well-ordered superstructure phases evolve which only depend on the amount of oxygen atoms at the surface. The coverage and consequently the superstructure can be adjusted by applying a different oxygen dosage or by desorption of oxygen upon annealing at specific temperatures between 1300 and $1500 \mathrm{~K}$. For coverages above one monolayer, this includes also the reversible reconstruction of the Mo(112) surface and the formation of molybdenum oxide layers. Maxima in the intensity of specularly scattered He atoms provided the optimal coverage for the different superstructure phases.

From fast atom diffraction in addition to size and symmetry of the surface unit cell, information on atomic positions was deduced. Evidence for a glide plane symmetry for the $p g(2 \times 1)$ phase results in the presence of two oxygen atoms in the unit cell and therefore a coverage of $\Theta=1 \mathrm{ML}$. So far, for this phase a coverage of $0.5 \mathrm{ML}$ was assumed and, consequently, most of the former atomic models in the low-coverage regime could be disproved. From periodic intensity variations of diffraction spots for the $c(2 \times 4)$ and $p g(2 \times 1)$ phases, the spacings of oxygen rows and therefore first information on adsorption sites were derived.

The detailed atomic positions of four superstructures with coverages $\Theta_{\mathrm{O}}=0.5-1.5 \mathrm{ML}$ were deduced by means of two types of triangulation techniques. Oxygen is mostly adsorbed at pseudo-threefold-hollow sites with two different bonding geometries, namely, bond to one topmost Mo atom and two atoms of the second Mo layer or vice versa. On the basis of stability calculations using DFT, none of these two geometries are favorable in energy [48]. This is supported experimentally since with increasing coverage the adsorption site changes from one geometry to the other and back. From the pronounced decay of specular intensity for the transitions from $c(2 \times 4)$ to $p g(2 \times 1)$ and to $c(4 \times 2)$ follows a substantial disorder during 
the change of adsorption sites. Fedorus et al. [54] observed a reduced work function for the $p(2 \times 1)$ phase and concluded a change of adsorption sites from short-bridge to hollow site. On the basis of our structure investigations, this may also be explained by the different hollow site geometries for $p g(2 \times 1)$.

For the transition from $c(4 \times 2)$ to $p(1 \times 2)$ with oxygen coverages 1.25 and $1.5 \mathrm{ML}$, respectively, both structures with similar adsorption sites coexist at the surface. The need of additional adsorption sites for coverages above $1 \mathrm{ML}$ supports substrate reordering with a loss of one molybdenum atom per $c(4 \times 2)$ unit cell. With increasing oxygen coverage and for sufficiently high temperatures $(T>700 \mathrm{~K})$, further adsorbate and substrate reordering results in the missing-row reconstruction with $p(1 \times 2)$ unit cell. The atomic positions derived for this superstructure agree very well with the result of DFT calculations from Sierka et al. $[48,60]$. In this respect, also calculations for the $c(4 \times 2)$ unit cell would be of interest to validate this phase as an intermediate step in the missing-row formation.

\section{ACKNOWLEDGMENTS}

This work was supported by the Deutsche Forschungsgemeinschaft in Sonderforschungsbereich 546 and under Contract No. Wi 1336. We thank D. Blauth, M. Reinhardt, K. Maass, and G. Lindenberg for their assistance in the preparation of the experiments.
[1] D. P. Woodruff, Modern Techniques of Surface Science (Cambridge University Press, Cambridge, 1994).

[2] M. A. Van Hove, Surf. Interface Anal. 28, 36 (1999).

[3] D. Woodruff, Surf. Sci. 500, 147 (2002).

[4] D. P. Woodruff, J. Phys.: Condens. Matter 22, 084016 (2010).

[5] K. Heinz and L. Hammer, J. Phys. Chem. B 108, 14579 (2004).

[6] K. Heinz, L. Hammer, and S. Müller, J. Phys.: Condens. Matter 20, 304204 (2008).

[7] H. Winter, Phys. Rep. 367, 387 (2002).

[8] R. Pfandzelter, T. Bernhard, and H. Winter, Phys. Rev. Lett. 90, 036102 (2003).

[9] T. Bernhard, J. Seifert, and H. Winter, J. Phys.: Condens. Matter 21, 134001 (2009).

[10] J. Seifert and H. Winter, Surf. Sci. 603, L109 (2009).

[11] H. Winter and A. Schüller, Prog. Surf. Sci. 86, 169 (2011).

[12] J. Seifert and H. Winter (unpublished).

[13] A. Mertens and H. Winter, Phys. Rev. Lett. 85, 2825 (2000).

[14] T. Bernhard and H. Winter, Nucl. Instrum. Methods Phys. Res., Sect. B 232, 16 (2005).

[15] A. Schüller, S. Wethekam, and H. Winter, Phys. Rev. Lett. 98, 016103 (2007).

[16] P. Rousseau, H. Khemliche, A. G. Borisov, and P. Roncin, Phys. Rev. Lett. 98, 016104 (2007).

[17] N. Bundaleski, H. Khemliche, P. Soulisse, and P. Roncin, Phys. Rev. Lett. 101, 177601 (2008).

[18] A. Schüller and H. Winter, Phys. Rev. Lett. 100, 097602 (2008).

[19] A. Schüller, M. Busch, J. Seifert, S. Wethekam, H. Winter, and K. Gärtner, Phys. Rev. B 79, 235425 (2009).

[20] J. Seifert, A. Schüller, H. Winter, R. Włodarczyk, J. Sauer, and M. Sierka, Phys. Rev. B 82, 035436 (2010).

[21] J. Seifert, M. Busch, E. Meyer, and H. Winter, Phys. Rev. Lett. 111, 137601 (2013).

[22] M. Debiossac, A. Zugarramurdi, P. Lunca-Popa, A. Momeni, H. Khemliche, A. G. Borisov, and P. Roncin, Phys. Rev. Lett. 112, 023203 (2014).

[23] F. Aigner, N. Simonovic, B. Solleder, L. Wirtz, and J. Burgdörfer, Phys. Rev. Lett. 101, 253201 (2008).

[24] J. R. Manson, H. Khemliche, and P. Roncin, Phys. Rev. B 78, 155408 (2008).

[25] N. Bundaleski, P. Soulisse, A. Momeni, H. Khemliche, and P. Roncin, Nucl. Instrum. Methods Phys. Res., Sect. B 269, 1216 (2011).
[26] J. Lienemann, A. Schüller, D. Blauth, J. Seifert, S. Wethekam, M. Busch, K. Maass, and H. Winter, Phys. Rev. Lett. 106, 067602 (2011).

[27] J. Seifert, A. Schüller, H. Winter, and K. Gärtner, Nucl. Instrum. Methods Phys. Res., Sect. B 269, 1212 (2011).

[28] M. Busch, J. Seifert, E. Meyer, and H. Winter, Phys. Rev. B 86 , 241402 (2012).

[29] A. Zugarramurdi and A. G. Borisov, Phys. Rev. A 86, 062903 (2012).

[30] A. Zugarramurdi and A. G. Borisov, Phys. Rev. A 87, 062902 (2013).

[31] A. Zugarramurdi, M. Debiossac, P. Lunca-Popa, L. S. Alarcon, A. Momeni, H. Khemliche, P. Roncin, and A. G. Borisov, Phys. Rev. A 88, 012904 (2013).

[32] M. Debiossac and P. Roncin, Phys. Rev. A 90, 054701 (2014).

[33] M. Debiossac, A. Zugarramurdi, H. Khemliche, P. Roncin, A. G. Borisov, A. Momeni, P. Atkinson, M. Eddrief, F. Finocchi, and V. H. Etgens, Phys. Rev. B 90, 155308 (2014).

[34] M. S. Gravielle and J. E. Miraglia, Phys. Rev. A 90, 052718 (2014).

[35] J. Seifert, J. Lienemann, A. Schüller, and H. Winter, Nucl. Instrum. Methods Phys. Res., Sect. B 350, 99 (2015).

[36] M. S. Gravielle and J. E. Miraglia, Phys. Rev. A 92, 062709 (2015).

[37] J. Seifert and H. Winter, Surf. Sci. 610, L1 (2013).

[38] P. Atkinson, M. Eddrief, V. H. Etgens, H. Khemliche, M Debiossac, A. Momeni, M. Mulier, B. Lalmi, and P. Roncin, Appl. Phys. Lett. 105, 021602 (2014).

[39] H. Khemliche, P. Rousseau, P. Roncin, V. H. Etgens, and F. Finocchi, Appl. Phys. Lett. 95, 151901 (2009).

[40] A. Schüller, M. Busch, S. Wethekam, and H. Winter, Phys. Rev. Lett. 102, 017602 (2009).

[41] A. Schüller, S. Wethekam, D. Blauth, H. Winter, F. Aigner, N. Simonović, B. Solleder, J. Burgdörfer, and L. Wirtz, Phys. Rev. A 82, 062902 (2010).

[42] J. Seifert and H. Winter, Phys. Rev. Lett. 108, 065503 (2012).

[43] A. Schüller, D. Blauth, J. Seifert, M. Busch, H. Winter, K. Gärtner, R. Włodarczyk, J. Sauer, and M. Sierka, Surf. Sci. 606, 161 (2012).

[44] J. Seifert and H. Winter, Nucl. Instrum. Methods Phys. Res., Sect. B 315, 9 (2013). 
[45] A. Zugarramurdi, M. Debiossac, P. Lunca-Popa, A. J. Mayne, A. Momeni, A. G. Borisov, Z. Mu, P. Roncin, and H. Khemliche, Appl. Phys. Lett. 106, 101902 (2015).

[46] F. E. Feiten, J. Seifert, J. Paier, H. Kuhlenbeck, H. Winter, J. Sauer, and H.-J. Freund, Phys. Rev. Lett. 114, 216101 (2015).

[47] J. Seifert, M. Busch, E. Meyer, and H. Winter, Phys. Rev. B 89, 075404 (2014).

[48] M. Sierka, T. K. Todorova, J. Sauer, S. Kaya, D. Stacchiola, J. Weissenrieder, S. Shaikhutdinov, and H. J. Freund, J. Chem. Phys. 126, 234710 (2007).

[49] S. Kaya, J. Weissenrieder, D. Stacchiola, T. K. Todorova, M. Sierka, J. Sauer, S. Shaikhutdinov, and H. J. Freund, Surf. Sci. 602, 3338 (2008).

[50] F. Besenbacher and J. K. Norskov, Prog. Surf. Sci. 44, 5 (1993).

[51] X. Duan, O. Warschkow, A. Soon, B. Delley, and C. Stampfl, Phys. Rev. B 81, 075430 (2010).

[52] K.-i. Fukui, T. Aruga, and Y. Iwasawa, Surf. Sci. 281, 241 (1993).

[53] T. Aruga, K. Tatenko, K.-i. Fukui, and Y. Iwasawa, Surf. Sci. 324, 17 (1995).

[54] A. G. Fedorus, A. A. Mitryaev, and A. G. Naumovets, Eur. Phys. J. B 85, 408 (2012).

[55] A. K. Santra, B. K. Min, and D. W. Goodman, Surf. Sci. 513, L441 (2002).

[56] T. Schroeder, J. Zegenhagen, N. Magg, B. Immaraporn, and H. J. Freund, Surf. Sci. 552, 85 (2004).

[57] K. Fukui, K. Motoda, and Y. Iwasawa, J. Phys. Chem. B 102, 8825 (1998)

[58] T. Sasaki, Y. Goto, R. Tero, K. Fukui, and Y. Iwasawa, Surf. Sci. 502-503, 136 (2002).

[59] A. Kiejna and R. M. Nieminen, J. Chem. Phys. 122, 044712 (2005).

[60] M. Sierka, Prog. Surf. Sci. 85, 398 (2010).

[61] DLD40 Delay-Line Detector System, RoentDek Handels GmbH, Kelkheim, Germany.

[62] E. Liénard, M. Herbane, G. Ban, G. Darius, P. Delahaye, D. Durand, X. Fléchard, M. Labalme, F. Mauger, A. Mery, O. Naviliat-Cuncic, and D. Rodríguez, Nucl. Instrum. Methods Phys. Res., Sect. A 551, 375 (2005).

[63] Passivated Implanted Planar Silicon detector (PIPS), Canberra Inc.

[64] F. Aumayr, G. Lakits, and H. Winter, Appl. Surf. Sci. 47, 139 (1991).

[65] D. S. Gemmell, Rev. Mod. Phys. 46, 129 (1974).

[66] A. W. Kleyn and T. C. M. Horn, Phys. Rep. 199, 191 (1991).
[67] A. Schüller, G. Adamov, S. Wethekam, K. Maass, A. Mertens, and H. Winter, Phys. Rev. A 69, 050901 (2004).

[68] R. Pfandzelter, T. Bernhard, and H. Winter, Surf. Rev. Lett. 10, 399 (2003); T. Bernhard, R. Pfandzelter, and H. Winter, Nucl. Instrum. Methods Phys. Res., Sect. B 219-220, 567 (2004); M. Busch, M. Gruyters, and H. Winter, Surf. Sci. 600, 4166 (2006).

[69] T. Bernhard, M. Baron, M. Gruyters, and H. Winter, Phys. Rev. Lett. 95, 087601 (2005).

[70] J. Seifert, T. Bernhard, M. Gruyters, and H. Winter, Phys. Rev. B 76, 224405 (2007).

[71] J. Seifert, D. Blauth, and H. Winter, Phys. Rev. Lett. 103, 017601 (2009).

[72] D. J. O'Connor and J. P. Biersack, Nucl. Instrum. Methods Phys. Res., Sect. B 15, 14 (1986).

[73] S. Wethekam and H. Winter, Nucl. Instrum. Methods Phys. Res., Sect. B 258, 48 (2007).

[74] J. Seifert, E. Meyer, H. Winter, and H. Kuhlenbeck, Surf. Sci. 606, L41 (2012).

[75] J. E. Valdés, P. Vargas, C. Celedón, E. Sánchez, L. Guillemot, and V. A. Esaulov, Phys. Rev. A 78, 032902 (2008).

[76] D. Farias and K.-H. Rieder, Rep. Prog. Phys. 61, 1575 (1998).

[77] J. van der Veen, Surf. Sci. Rep. 5, 199 (1985).

[78] H. Niehus, W. Heiland, and E. Taglauer, Surf. Sci. Rep. 17, 213 (1993).

[79] See Supplemental Material at http://link.aps.org/supplemental/ 10.1103/PhysRevB.93.205417 for details about structure determination for monolayer silica on $\mathrm{Mo}(112)$ using secondary electron based triangulation.

[80] T. K. Todorova, M. Sierka, J. Sauer, S. Kaya, J. Weissenrieder, J.-L. Lu, H.-J. Gao, S. Shaikhutdinov, and H.-J. Freund, Phys. Rev. B 73, 165414 (2006); S. Kaya, M. Baron, D. Stacchiola, J. Weissenrieder, S. Shaikhutdinov, T. Todorova, M. Sierka, J. Sauer, and H.-J. Freund, Surf. Sci. 601, 4849 (2007); S. Shaikhutdinov and H. J. Freund, Adv. Mater. 25, 49 (2013).

[81] T. Schroeder, J. B. Giorgi, A. Hammoudeh, N. Magg, M. Baumer, and H. J. Freund, Phys. Rev. B 65, 115411 (2002).

[82] U. Specht, M. Busch, J. Seifert, A. Schüller, H. Winter, K. Gärtner, R. Wlodarczyk, M. Sierka, and J. Sauer, Phys. Rev. B 84, 125440 (2011).

[83] Q. Liu, L. Li, N. Cai, W. A. Saidi, and G. Zhou, Surf. Sci. 627, 75 (2014).

[84] N. Wu, Y. B. Losovyj, Z. Yu, R. F. Sabirianov, W. N. Mei, N. Lozova, J. A. C. Santana, and P. A. Dowben, J. Phys.: Condens. Matter 21, 474222 (2009). 